\title{
JAZF1-SUZ12 dysregulates PRC2 function and gene expression during cell differentiation
}

\section{Manuel Tavares' ${ }^{1}$, Garima Khandelwal ${ }^{1}$, Joanne Mutter ${ }^{2}$, Keijo Viiri' ${ }^{1,3}$, Manuel Beltran ${ }^{1,4}$,}

5 Jan J. Brosens², Richard G. Jenner ${ }^{1,5}$

${ }^{1}$ UCL Cancer Institute and Cancer Research UK UCL Centre, University College London (UCL), London, WC1E 6BT, UK.

${ }^{2}$ Warwick Medical School, Division of Biomedical Sciences, University of Warwick, Coventry 10 CV4 7AL, UK

${ }^{3}$ Current address: Faculty of Medicine and Health Technology, Tampere University, Tampere, Finland.

${ }^{4}$ Current address: Department of Biology and Biotechnology Charles Darwin, Sapienza University 15 of Rome, Rome, Italy.

\footnotetext{
${ }^{5}$ Correspondence: r.jenner@ucl.ac.uk
} 


\section{ABSTRACT}

25 Polycomb repressive complex 2 (PRC2) methylates histone H3 lysine 27 (H3K27me3) to maintain repression of genes specific for other cell types and is essential for cell differentiation. In endometrial stromal sarcoma, the PRC2 subunit SUZ12 is often fused with the NuA4/TIP60 subunit JAZF1. Here, we show that JAZF1-SUZ12 dysregulates PRC2 composition, recruitment, histone modification, gene expression and cell differentiation. The loss of the SUZ12 N-terminus

30 in the fusion protein disrupted interaction with the PRC2 accessory factors JARID2, EPOP and PALI1 and prevented recruitment of PRC2 from RNA to chromatin. In undifferentiated cells, JAZF1-SUZ12 occupied PRC2 target genes but gained a JAZF1-like binding profile during cell differentiation. JAZF1-SUZ12 reduced H3K27me3 and increased H4Kac at PRC2 target genes, and this was associated with disruption in gene expression and cell differentiation programs. These

35 results reveal the defects in chromatin regulation caused by JAZF1-SUZ12, which may underlie its role in oncogenesis. 


\section{INTRODUCTION}

Endometrial stromal tumours are a set of uterine malignancies that are divided into four subtypes; benign endometrial stromal nodules (ESN), low-grade endometrial stromal sarcoma (LG-ESS),

45 high-grade endometrial stromal sarcoma (HG-ESS) and undifferentiated uterine sarcoma (UUS). Around 50\% of ESN and LG-ESS cases exhibit the chromosomal rearrangement $\mathrm{t}(7 ; 17)(\mathrm{p} 15: \mathrm{q} 21)$ (Chiang et al., 2011; Micci et al., 2016), which results in production of a JAZF1-SUZ12 fusion protein that comprises the first 128 amino acids of JAZF1 in place of the first 93 amino acids of SUZ12 (Koontz et al., 2001). The WT SUZ12 allele is also silenced in these LG-ESS cases (Li et al 2007). $40 \%$ of LG-ESS patients do not respond to current therapeutic regimens, highlighting the need for greater understanding of JAZF1-SUZ12 function and the development of new treatment strategies for this disease (Amant et al., 2007; Beck et al., 2012) .

SUZ12 is a core subunit of polycomb repressive complex 2 (PRC2), along with EZH2 or EZH1,

55 EED and RBBP4 or RBBP7 (Margueron and Reinberg, 2011). PRC2 associates with genes encoding developmental regulators specific for other cell types or cell differentiation stages (Azuara et al., 2006; Boyer et al., 2006; Bracken et al., 2006; Lee et al., 2006). At these genes, PRC2 trimethylates histone H3 lysine 27 (H3K27me3), which allows binding of the canonical form of PRC1 and formation of a repressive chromatin structure that maintains genes in a repressed 60 state. Mice lacking Suz12 die during embryogenesis due to defects in gastrulation (Pasini et al., 2004; Lee et al., 2006), phenocopying Ezh2 or Eed-null mutants (Faust et al, 1995; O'Carroll et al, 2001). This role for SUZ12 in early development is reflected by its requirement for embryonic stem cell (ESC) differentiation in vitro (Lee et al., 2006; Pasini et al., 2007; Riising et al., 2014). 
PRC2 is also required for the differentiation of numerous other cell types, both during

65 embryogenesis and throughout life, and is frequently dysregulated in cancer (Comet et al., 2016;

Deevy \& Bracken, 2019; Prezioso \& Orlando, 2011; Schuettengruber et al., 2017).

In addition to the core PRC2 subunits, a number of accessory factors have been discovered that define two variants of PRC2; PRC2.1 and PRC2.2 (Alekseyenko et al, 2014; Beringer et al., 2016;

70 Conway et al., 2018; Grijzenhout et al., 2016; Hauri et al., 2016; Liefke et al, 2016). PRC2.1 is composed of the PRC2 core plus one of PCL1, PCL2 or PCL3 (also named PHF1, MTF2 and PHF19) along with either EPOP (Alekseyenko et al., 2014; Beringer et al., 2016; Liefke et al., 2016; Zhang et al., 2011), PALI1 or PALI2 (Alekseyenko et al., 2014; Conway et al., 2018; Zhang et al., 2011). PRC2.2 is less complex and is formed by the PRC2 core plus AEBP2 and JARID2

75 (Alekseyenko et al., 2014; Grijzenhout et al., 2016; Hauri et al., 2016). The accessory subunits act in combination to recruit PRC2 to its target sites (Højfeldt et al., 2018; Højfeldt et al., 2019; Oksuz et al., 2018; Perino et al., 2018; Wang et al., 2017a; Youmans et al., 2018) and are essential for normal development (Brien et al., 2012; Conway et al., 2018; Grijzenhout et al., 2016; Landeira et al., 2010; Li et al., 2010, 2017; Liefke et al., 2016; Shen et al., 2009; Walker et al., 2010; Zhang et al., 2011). SUZ12 interacts with EZH2 and EED through its C-terminal VEFS domain, and with JARID2, AEBP2, EPOP and PCL proteins through the N-terminal part of the protein (Chen et al., 2018; Kasinath et al., 2018; Kloet et al., 2016; Youmans et al., 2018). Recently, an additional factor, CXorf67/EZHIP/CATACOMB, was discovered to interact with core PRC2 and inhibit its histone methyltransferase activity (Hübner et al., 2019; Jain et al., 2019; Piunti et al., 2019; Ragazzini et al., 2019). 
In addition to binding to chromatin, PRC2 also interacts with RNA. First found to bind specific long non-coding RNAs, PRC2 has since been found to primarily interact with nascent pre-mRNA in cells (Beltran et al., 2016; Davidovich et al., 2013; Hendrickson et al., 2016; Kaneko et al.,

90 2013; Zhao et al., 2010), with a preference for G-quadruplex-forming G-tract repeats (Beltran et al., 2019; Wang et al., 2017b). In vitro, G-quadruplex RNA inhibits the interaction of PRC2 with DNA, nucleosomes and H3 tails (Beltran et al., 2016, Beltran et al., 2019; Long et al., 2017; Wang et al., 2017a; Zhang et al., 2019) and, in ESC, transcriptional inhibition (Riising et al., 2014), polyA-site insertion (Kaneko et al., 2014), and RNA degradation (Beltran et al., 2016) induce

95 PRC2 recruitment to active genes. Reciprocally, blocking nuclear RNA degradation (Garland et al., 2019) or tethering G-tract RNAs to polycomb target genes removes PRC2 from chromatin (Beltran et al., 2019), supporting a model in which nascent RNA restricts PRC2 recruitment to genes that are already silent (Kaneko et al., 2014, Beltran et al., 2016; Comet et al., 2016; Hosogane et al., 2016; Riising et al., 2014) .

100

The effect of JAZF1-SUZ12 on PRC2 function is not well understood. Ectopic expression of JAZF1-SUZ12 and knockdown of endogenous SUZ12 in HEK293 cells increased cell proliferation and resistance to hypoxia (Li et al., 2007). JAZF1 fusion has been reported to reduce interaction of SUZ12 with EZH2 and EED and consequently reduce PRC2 H3K27

105 methyltransferase activity (Ma et al., 2016). JAZF1-SUZ12 has also been found to lack interaction with JARID2 and EPOP (Chen et al., 2018) but its interaction with other PRC2 accessory factors has not been measured. 
Compared to SUZ12, understanding of JAZF1 function has been limited. JAZF1 interacts with the

110 orphan nuclear receptor NR2C2 (TAK1/TR4), inhibiting its activity (Nakajima et al., 2004) and regulates genes with functions in translation and RNA splicing (Kobiita et al., 2020; Procida et al., 2021). Shedding more light on the mechanism of JAZF1 action, the protein was recently found to be associated with the NuA4/TIP60 histone acetyltransferase complex (Piunti et al., 2019; Procida et al., 2021) which exchanges H2A for H2A.Z (Nishibuchi et al., 2014; Xu et al., 2012) and

115 acetylates histone $\mathrm{H} 4$ (H4Kac), as well as histone H2A and its variants (Steunou et al., 2014). Correspondingly, fusion of JAZF1-SUZ12 results in ectopic interaction between PRC2 and NuA4 components (Piunti et al., 2019). The association of JAZF1 with NuA4 is consistent with reports of other fusion events between PRC2 and NuA4 subunits in LG-ESS, namely EPC1-PHF1 (Micci et al., 2006), JAZF1-PHF1 (Panagopoulos et al., 2008), MEAF6-PHF1(Panagopoulos et al., 2012),

120 MBTD1-CXorf67/EZHIP (Dewaele et al., 2014), BRD8-PHF1(Davidson and Micci, 2017), MEAF6-SUZ12 (Makise et al., 2019) and MBTD1-PHF1 (Han et al., 2020), and indicates that the fusion of PRC2 with NuA4 drives oncogenesis in all of these cases. However, how JAZF1-SUZ12 affects PRC2 composition, recruitment and patterns of $\mathrm{H} 3 \mathrm{~K} 27 \mathrm{me} 3$ and the impact of these changes on gene expression and cell differentiation remain unclear.

Here, we show that JAZF1-SUZ12 disrupts interaction of PRC2 with JARID2, EPOP and PALI1, and dysregulates PRC2 recruitment to chromatin. We also separate changes due to loss of the SUZ12 N-terminus from those caused by fusion to JAZF1. JAZF1-SUZ12 chromatin occupancy diverges from WT SUZ12 during cell differentiation and resulted in reduced H3K27me3 and 130 increased H4Kac at PRC2 target genes. ESC expressing JAZF1-SUZ12 show altered patterns of gene expression and disrupted embryoid body (EB) formation and JAZF1-SUZ12 also drives gene 
expression changes in primary human endometrial stromal cells (hEnSC). We propose that these effects contribute to JAZF1-SUZ12-driven oncogenesis.

\section{RESULTS}

\section{JAZF1-SUZ12 lacks interaction with JARID2, EPOP and PALI1 due to loss of the SUZ12 N-terminus}

We first sought to establish the effect of JAZF1 fusion with SUZ12 on PRC2 composition. To

140 address this, we generated Suz12 ${ }^{\mathrm{GT} / \mathrm{GT}}$ ESC lines stably expressing FLAG-tagged SUZ12, JAZF1SUZ12, JAZF1 or GFP (Figure 1A). To distinguish the changes in PRC2 composition due to loss the of the N-terminal 93 amino acids of SUZ12 and the changes due to gain of the first 128 amino acids of JAZF1, we also generated a cell line stably expressing FLAG-tagged SUZ12 $\Delta 93$. Immunoblotting confirmed that the levels of SUZ12, SUZ12 $\triangle 93$ and JAZF1-SUZ12 proteins were 145 similar to each other and to endogenous SUZ12 (Figure S1A).

We then measured the effect of SUZ12 $\triangle 93$ and JAZF1-SUZ12 on PRC2 composition by coimmunoprecipitation and immunoblotting (Figure 1B). Co-immunoprecipitation of endogenous SUZ12 from WT ESC provided an additional control. This revealed that SUZ12, SUZ12 $\triangle 93$ and

JAZF1-SUZ12 interacted with the core subunit EZH2 and the accessory subunits AEBP2 and PCL2. However, unlike SUZ12, SUZ12 493 and JAZF1-SUZ12 did not interact with JARID2 or EPOP. These findings were confirmed by reciprocal co-IPs of tagged forms of AEBP2, EPOP and PCL3 (Figure S1B-D). 
155 To determine the effect of JAZF1-SUZ12 on the association of PALI1 with PRC2, we cotransfected FS2-tagged SUZ12, SUZ12 493 , JAZF1-SUZ12 and JAZF1 constructs with HA/FLAG-tagged PALI1 and performed Strep-Tactin affinity purification (Figure 1C). We found that PALI1 did not interact with SUZ12 493 or JAZF1-SUZ12, thus following the same pattern as EPOP and JARID2.

We also sought to confirm recent findings that JAZF1-SUZ12 exhibited an ectopic interaction with the NuA4/TIP60 complex (Piunti et al., 2019) We found that IP for JAZF1-SUZ12 or JAZF1 coprecipitated the NuA4 subunit TRRAP but that this wasn't the case for IP of SUZ12 or SUZ12 $\triangle 93$

(Figure 1B). Therefore, we conclude that fusion of SUZ12 with JAZF1 disrupts interaction with 165 EPOP, JARID2 and PALI1 due to loss of the SUZ12 N-terminus and induces interaction of SUZ12 with TRRAP due to gain of the N-terminus of JAZF1.

\section{Lack of recruitment of JAZF1-SUZ12 to chromatin upon RNA depletion due to loss of interaction with JARID2 and EPOP}

170 The recruitment of PRC2 to chromatin is antagonised by RNA and this can be observed by the increase in PRC2 chromatin association upon RNA degradation in cells (Beltran et al., 2016, Beltran et al., 2019). Thus, we sought to determine the effect of JAZF1-SUZ12 on the recruitment of PRC2 to chromatin upon RNaseA treatment. ESC expressing SUZ12, SUZ12 493 or JAZF1SUZ12 were permeabilised, mock-treated or treated with RNaseA and the nucleoplasmic and 175 chromatin fractions purified. This confirmed that RNaseA treatment increased the association of WT SUZ12 with chromatin but decreased the association of FUS with chromatin (Figure 2A), as previously described (Beltran et al., 2016). In contrast, SUZ12 $\triangle 93$ and JAZF1-SUZ12 were both 
depleted from chromatin by RNaseA treatment, as was EZH2 in cells expressing these proteins. Thus, loss of the SUZ12 N-terminus prevents the recruitment of JAZF1-SUZ12 from RNA to chromatin.

Given that SUZ12 993 and JAZF1-SUZ12 lack interaction with JARID2, EPOP and PALI1, we asked whether this was the reason that these proteins were not recruited to chromatin upon RNA degradation. We first tested the effect of loss of the PRC2.2 subunits AEBP2 or JARID2 on PRC2 185 chromatin association upon RNA degradation. Aebp $2^{\mathrm{WT} / \mathrm{WT}}$, Aebp $2^{\mathrm{GT} / \mathrm{GT}}$ and Jarid2 $2^{\mathrm{GT} / \mathrm{GT}}$ cells were permeabilized, mock or RNaseA-treated and fractionated as before (Figure 2B). We found that loss of AEBP2 had no effect on the recruitment of SUZ12 and EZH2 to chromatin upon RNA depletion. In contrast, loss of JARID2 abrogated the recruitment of PRC2 subunits to chromatin (Figure 2B). These results show that within PRC2.2, JARID2 is necessary PRC2 recruitment from 190 RNA to chromatin, while AEBP2 is not.

We next determined the requirement for the PRC2.1 subunits EPOP, PALI1 and PCL1-3 on the recruitment of PRC2 to chromatin upon RNA depletion. In cells lacking EPOP, we found there was no enrichment of SUZ12 and EZH2 in the chromatin fraction upon RNaseA treatment (Figure

195 2C). In contrast, we did not observe a change in the recruitment of SUZ12 to chromatin in cells lacking PALI1 or PCL1-3 (Figures S2A and S2B). We conclude that JAZF1-SUZ12-containing PRC2 is not recruited from RNA to chromatin and that this is due to lack of interaction with JARID2 and EPOP. 


\section{JAZF1-SUZ12 takes on a JAZF1-like binding profile during cell differentiation}

Considering that JAZF1-SUZ12 displayed defects in recruitment from RNA to chromatin, we next asked how fusion to JAZF1 affected the pattern of SUZ12 chromatin occupancy during cell differentiation. To measure this, we performed calibrated ChIP-seq for FLAG-tagged GFP, SUZ12, SUZ12 493 , JAZF1-SUZ12 and JAZF1 in ESC and at 4 and 8 days after induction of EB formation. We first identified binding sites for each protein at each timepoint and used hierarchical clustering to determine how these were related to one another (Figure 3A). This showed that SUZ12 and JAZF1 samples clustered separately, demonstrating they had distinct binding profiles. SUZ12 $\triangle 93$ samples clustered together with SUZ12 indicating that loss of SUZ12 N-terminus did

210 not completely disrupt the pattern of PRC2 chromatin occupancy. Strikingly, the JAZF1-SUZ12 binding profile resembled SUZ12 and SUZ12 $\triangle 93$ in ESC but in EBs, its binding profile was more similar to that of JAZF1. This shift of JAZF1-SUZ12 towards a JAZF1 binding profile during ESC differentiation was also apparent by plotting SUZ12 and JAZF1 occupancy at JAZF1-SUZ12 binding sites in ESC compared with JAZF1-SUZ12 binding sites in EBs (Figure 3B). Indeed,

215 quantifying the number of binding sites shared between the different factors revealed that JAZF1SUZ12 occupied $39 \%$ of JAZF1 binding sites in ESC, which increased to $82 \%$ of JAZF1 binding sites in day 8 EBs (Figure S3A). Our analysis also revealed that JAZF1-SUZ12 occupied a large number of sites that were not shared with SUZ12 or JAZF1 and that the number of these ectopic binding sites also increased during ESC differentiation (Figure S3A).

We also assessed how the changes in SUZ12 genome occupancy that occur during differentiation were affected by fusion to JAZF1. Comparing SUZ12 and SUZ12 $\triangle 93$ occupancy between ESC and EBs demonstrated that these proteins tended to be depleted from their target genes during 
differentiation (Figure S3B), as previously observed for endogenous PRC2 (Kloet et al., 2016). In

225 contrast, JAZF1-SUZ12 tended to be retained at its target genes during ESC differentiation.

These differences JAZF1-SUZ12 and SUZ12 occupancy could also be observed at individual genes (Figures 3C and S3C). At genes with broad regions of SUZ12 binding, for example the Hoxa cluster, Foxal or Pax3, SUZ12 and JAZF1-SUZ12 occupancy was similar, with extensive binding

230 in ESC that decreased during differentiation. At genes with more narrow regions of SUZ12 occupancy, for example $T$ (Brachyury), Bmp6 or Fgf5, JAZF1-SUZ12 binding was greatly reduced. This was also the case for SUZ12 $\triangle 93$, indicating that the reduction in JAZF1-SUZ12 binding at these sites was due to lack of the SUZ12 N-terminus. In contrast, JAZF1-SUZ12 binding could be observed at other genes, such as Bax, Cbx3 and Mcl1, that lacked SUZ12 occupancy but

235 were instead occupied by JAZF1. At these genes, JAZF1-SUZ12 occupancy increased during ESC differentiation. Finally, sites occupied by JAZF1-SUZ12 but not SUZ12, SUZ12 293 or JAZF1 could be identified, for example a region upstream of Gata4 (Figure 3C), while other sites from which SUZ12 and SUZ12 $\triangle 93$ were depleted during differentiation instead retained JAZF1SUZ12, for example $\operatorname{Prdm} 8$ and Hhex (Figure S3C).

We conclude from these analyses that loss of the SUZ12 N-terminus reduces JAZF1-SUZ12 recruitment to PRC2 target genes, especially those with narrower regions of occupancy, and that fusion to JAZF1 triggers recruitment to JAZF1 target sites and to an additional set of sites that lack SUZ12 or JAZF1 occupancy. 
To determine how these changes in chromatin occupancy might impact cell state, we used Gene Ontology to identify functional terms enriched in the sets of genes associated with SUZ12, JAZF1SUZ12, or JAZF1 binding sites (Figure 3D and Table S1). We found that some functional annotations, for example those related to development and transcription factor activity, were

250 shared between the sets of genes occupied by SUZ12 and JAZF1-SUZ12, but not by genes occupied by JAZF1. In contrast, other functional gene classes targeted by SUZ12, for example genes with functions in cell adhesion and chemotaxis, were lost from the set of genes occupied by JAZF1-SUZ12, either in both ESC and day 8 EBs or only in EBs. This is consistent with reduced binding of JAZF1-SUZ12 to some SUZ12 target sites (Figures 3C and S3C). Instead of these gene

255 functions, JAZF1-SUZ12 target genes exhibited functions in common with genes bound by JAZF1, including terms related to cell metabolism, cellular localisation and cell cycle, and also functions that were specific to JAZF1-SUZ12 target genes, such as cytoskeletal protein binding and regulation of cell size. Thus, fusion to JAZF1 alters SUZ12 chromatin occupancy and this changes the functional classes of genes targeted by the protein.

\section{JAZF1-SUZ12 reduces H3K27me3 and increases H4Kac at PRC2 target genes}

We next considered the effect of JAZF1-SUZ12 on H3K27me3, catalysed by PRC2, and H4Kac, catalysed by NuA4/TIP60. To do this, we performed calibrated ChIP-seq for H3K27me3 and panH4Kac in ESC and EBs expressing WT SUZ12, SUZ12 993 or JAZF1-SUZ12. We first examined

265 the effect of JAZF1-SUZ12 on H3K27me3 at canonical PRC2 target sites. To allow measurement of the effect of JAZF1-SUZ12 on H3K27me3 separately from its effect on PRC2 occupancy, we plotted H3K27me3 at binding sites shared by SUZ12, SUZ12 293 and JAZF1-SUZ12 (Figure 4A). We found that ESC expressing JAZF1-SUZ12 exhibited lower levels of H3K27me3 at PRC2 target 
sites than cells expressing WT SUZ12. Examining individual genes revealed a reduction in

270 H3K27me3 even at loci with a broad region of SUZ12 binding at which JAZF1-SUZ12 occupancy was maintained, such as the Hoxa cluster, Foxal and Pax3 (Figures 4B and S4A). At genes with more narrow regions of SUZ12 occupancy from which JAZF1-SUZ12 binding was lost, such as T, Bmp6 and Fgf5, H3K27me3 was completely abrogated in ESC expressing JAZF1-SUZ12 (Figures 4B and S4A). This was also observed in cells expressing SUZ12 $\Delta 93$, indicating that the

275 reduction in $\mathrm{H} 3 \mathrm{~K} 27 \mathrm{me} 3$ at PRC2 target sites in cells expressing JAZF1-SUZ12 was due to loss of the SUZ12 N-terminus. Thus, we conclude that one way in which fusion of JAZF1 to SUZ12 alters chromatin state is by reducing $\mathrm{H} 3 \mathrm{~K} 27 \mathrm{me} 3$ at PRC2 target sites.

We also asked whether ectopic JAZF1-SUZ12 binding at non-PRC2 target sites, for example those 280 at Bax, Cbx3 and Mcl1, resulted in ectopic H3K27me3 (Figures 4B, S4A and S4B). However, we found that this was not the case and that $\mathrm{H} 3 \mathrm{~K} 27 \mathrm{me} 3$ remained low at these sites in cells expressing JAZF1-SUZ12, consistent with the limited amounts of H3K27me3 deposited by this protein at PRC2 target sites.

285 We next turned our attention to the effects of JAZF1-SUZ12 on H4Kac at PRC2 target sites. We observed an increase in H4Kac at PRC2 binding sites in ESC expressing JAZF1-SUZ12 and this was particularly noticeable at regions of broad SUZ12 occupancy (Figures 4A, 4B and S4A). Cells expressing SUZ12 $\triangle 93$ also exhibited an increase in H4Kac at these sites, suggesting that much of this effect was due to loss of the repressive function of $\mathrm{H} 3 \mathrm{~K} 27 \mathrm{me} 3$ rather than due to recruitment 290 of NuA4/TIP60. However, in EBs, JAZF1-SUZ12 caused a marked increase in H4Kac at PRC2 target sites that was not observed for SUZ12 $\triangle 93$, indicating this was due to fusion to JAZF1 
(Figure 4A). Increases in H4Kac in EBs expressing JAZF1-SUZ12 were also observed at individual genes including T, Pax3, Fgf5, Prdm8 and Hhex (Figures 4B and S4A). We did not observe an increase in H4Kac at JAZF1-SUZ12 binding sites shared with JAZF1 (Figure S4B),

295 likely because of endogenous NuA4/TIP60 activity at these sites. We conclude that, in addition to reducing H3K27me3, JAZF1-SUZ12 also increased the active histone mark H4Kac at PRC2 target genes, especially in differentiated cells.

\section{JAZF1-SUZ12 disrupts gene expression during cell differentiation}

300 We considered that changes in the pattern of PRC2 recruitment and histone modification induced by JAZF1-SUZ12 may be associated with alterations in the pattern of gene expression during ESC differentiation. To address this, we harvested RNA from Suz12 $2^{\mathrm{GT} / \mathrm{GT}}$ ESC stably expressing GFP, SUZ12, SUZ12 $\triangle 93$ or JAZF1-SUZ12 before and 4 and 8 days after induction of EB formation. We first determined whether JAZF1-SUZ12 had any effect on the expression of pluripotency 305 genes. We found that Oct4, Fgf4, Nanog and Utfl were repressed during EB formation to a similar extent in all of the cell lines, although for Oct4 and Fgf4, repression was delayed in cells containing SUZ12 $\triangle 93$ or JAZF1-SUZ12 (Figure 5A). These data indicate that the fusion of JAZF1 to SUZ12 has minor effects on silencing of the ESC gene expression program and that this effect is due to loss of the SUZ12 N-terminus.

Given that we had observed dysregulation of SUZ12 recruitment and histone modification during ESC differentiation, we next assessed the effect of JAZF1-SUZ12 on gene induction during EB formation (Figure 5B). We found that JAZF1-SUZ12 disrupted normal patterns of gene induction, but in different ways for different genes. Unlike cells expressing WT SUZ12, cells expressing 
315 JAZF1-SUZ12 did not induce Pax3 during differentiation and also exhibited reduced induction of Fgf5 and T. In contrast, Gata4 was induced more strongly during differentiation of cells expressing JAZF1-SUZ12. SUZ12 493 had similar effects to JAZF1-SUZ12 on T and Gata4 induction, indicating these changes were caused by loss of the SUZ12 N-terminus, whereas the effects of SUZ12 493 on $P a x 3$ and Fgf5 were distinct to those of JAZF1-SUZ12, indicating these changes

320 were caused by fusion to JAZF1.

We next considered whether these alterations in gene induction in cells expressing JAZF1-SUZ12 were associated with changes in EB formation. To address this, we induced differentiation of WT ESC and $\mathrm{Suz} 12^{\mathrm{GT} / \mathrm{GT}}$ ESC expressing GFP, SUZ12, SUZ12 $\Delta 93$ or JAZF1-SUZ12 and measured

325 the size of the resulting EBs after 2, 4 and 8 days (Figures 5C and D). We found that all cell lines formed EBs and, except for cells expressing GFP, these EBs increased in size between days 2 and 8, indicating some rescue of PRC2 function. Strikingly, EBs formed by cells expressing JAZF1SUZ12 were significantly larger than EBs formed by WT ESC or Suz12 ${ }^{\mathrm{GT} / \mathrm{GT}}$ ESC expressing the other constructs. Furthermore, the increase in the size of EBs formed from cells expressing JAZF1-

330 SUZ12 was associated with the formation of multiple cystic cavities (Figure 5D). Production of cystic cavities is associated with expression of endoderm markers such as Gata4 (Fujikura et al., 2002; Kulinski et al., 2015), consistent with enhanced expression of this gene in EBs containing JAZF1-SUZ12. We conclude that JAZF1-SUZ12 dysregulates gene expression and that this disrupts normal patterns of cell differentiation.

\section{JAZF1-SUZ12 alters gene expression in primary human endometrial stromal cells}

Given that JAZF1-SUZ12 altered gene expression in ESC, we asked whether this effect was also evident in the cell type in which the fusion event occurs; human endometrial stromal cells (hEnSC). 
To assess this, we transduced primary hEnSC with retroviral vectors encoding SUZ12, JAZF1-

340 SUZ12 or JAZF1, selected for cells expressing the transgenes, and purified RNA before and 4 and 8 days after induction of decidualisation with cyclic AMP (cAMP) and methyl-progesterone acetate (MPA) (Gellersen and Brosens, 2014) (Figures S5A and 5B). By day 8, each hEnSC culture changed from a fibroblast to an epithelioid phenotype and induced expression of the decidualisation marker PRL (Figures 6A and S5C). However, at day 0, we found that hEnSC

345 expressing JAZF1-SUZ12 already exhibited increased expression of IGFBP2 and ROR2 (Figure 6A), genes that have previously been linked to uterine sarcomas (Cuppens et al., 2015; Przybyl et al., 2018). Thus, JAZF1-SUZ12 disrupts gene expression in primary hEnSC as well as in ESC.

\section{DISCUSSION}

We have determined the effect of JAZF1-SUZ12 on PRC2 composition, recruitment, histone modification, gene expression and cell differentiation and identified the effects caused by loss of the SUZ12 N-terminus versus those caused by fusion to JAZF1. JAZF1-SUZ12 does not interact with the PRC2.1 subunits EPOP and PALI1 or the PRC2.2 subunit JARID2 and this prevents

355 recruitment from RNA to chromatin. We also confirm that JAZF1-SUZ12 interacts with the NuA4/TIP60 component TRRAP. These alterations in protein interactions are reflected in changes in JAZF1-SUZ12 chromatin occupancy during cell differentiation. In undifferentiated ESC, the pattern of JAZF1-SUZ12 chromatin occupancy resembles WT SUZ12 but becomes more similar to JAZF1 as ESC differentiate into EBs. JAZF1-SUZ12 reduces H3K27me3 and increases H4Kac 360 at PRC2 target genes, and this is coupled with changes in gene expression and morphology during ESC differentiation. JAZF1-SUZ12 also alters gene expression in primary hEnSC. Thus, we 
conclude that JAZF1-SUZ12 exhibits both loss and gain of function, which leads to dysregulation of gene expression and alterations in cell differentiation (Figure 6B). We propose that these effects of JAZF1-SUZ12 contribute to oncogenesis in cases of LG-ESS with the $t(7 ; 17)(\mathrm{p} 15: \mathrm{q} 21)$

365 rearrangement.

Our results confirm previous observations that JAZF1-SUZ12 does not interact with JARID2 or EPOP (Chen et al., 2018) and reveal that this is due to loss of the first 93 amino acids of SUZ12 rather than gain of the JAZF1 N-terminus. The requirement for the first 93 amino acids of SUZ12

370 for interaction with these proteins is reflected in cryo EM-based reconstructions of PRC2 structure that show that the SUZ12 ZnB (amino acids 76-110) and Zn (amino acids 420-500) regions come together to form a neck-like structure that interacts with JARID2 and is also consistent with the competition observed between JARID2 and EPOP for SUZ12 binding (Chen et al., 2018). We also show that deletion of the N-terminal 93 amino acids abrogates the interaction of SUZ12 with

375 PALI1. That SUZ12 interacts with PALI1 and EPOP through the same region is consistent with the mutual exclusive association of PRC2 with these proteins (Alekseyenko et al., 2014; Hauri et al., 2016). Similarly, that SUZ12 interacts with PALI1 and JARID2 through the same region is consistent with recent findings that PALI1 mimics the allosteric activation function of JARID2 (Zhang et al., 2020).

We found that JAZF1-SUZ12 and SUZ12 $\triangle 93$ are not recruited to chromatin upon RNA degradation and that this defect is recapitulated in cells lacking JARID2 or EPOP. This suggests that these factors are necessary for the recruitment of PRC2 from RNA to chromatin that occurs upon gene repression (Beltran et al., 2019). We have previously shown that JARID2 is not required 
385 for PRC2 RNA binding in cells (Beltran et al., 2016), suggesting that the lack of chromatin recruitment upon RNA degradation reflects a failure in transfer from RNA to chromatin rather than an initial lack of RNA binding. It is interesting that PCL2 is not required for PRC2 recruitment to chromatin upon RNA degradation, even though loss of PCL2 hugely diminishes steady state PRC2 chromatin association (Healy et al., 2019; Højfeldt et al., 2019; Li et al., 2017; Perino et al.,

390 2018). These results suggest that the interaction of PRC2 with chromatin that occurs upon release from RNA is mediated through a different mechanism that relies on JARID2 and EPOP.

Our data reveal how fusion to JAZF alters SUZ12 chromatin occupancy. We found that JAZF1SUZ12 exhibited a similar binding profile to WT SUZ12 in ESC but as cells differentiate, it takes 395 on a binding profile more similar to that of JAZF1. Thus, fusion of SUZ12 to JAZF1 has a bigger impact on genome localisation in differentiated cells than in undifferentiated cells. This switch in binding preference was not observed for SUZ12 $\triangle 93$, demonstrating that it depends on fusion to JAZF1 rather than loss of interaction with PRC2 accessory factors. These data are consistent with a model in which cell differentiation is accompanied by changes in chromatin state or changes in 400 the expression of PRC2 and/or NuA4 subunits that shifts the balance of JAZF1-SUZ12 from favouring PRC2 target sites to favouring JAZF1 target sites. We also found that JAZF1-SUZ12 occupied a set of sites that were not bound by SUZ12 or JAZF1 and the increase in the number of these ectopic binding sites during ESC differentiation reflected retention of JAZF1-SUZ12 at sites from which SUZ12 was normally lost.

SUZ12 $\triangle 93$ also displayed differences in binding and H3K27me3 activity compared with WT SUZ12. The effect of JARID2 (Healy et al., 2019; Højfeldt et al., 2019; Landeira et al., 2010; 
Oksuz et al., 2018), EPOP (Beringer et al., 2016) or PALI1 (Conway et al., 2018) deficiency on PRC2 genome occupancy or H3K27me3 has been measured previously but the effect of 410 simultaneous loss of all of these interactions provides a comprehensive view of the role of the SUZ12 ZnB/Zn region in PRC2 recruitment and activity. Although JAZF1-SUZ12 and SUZ12 93 binding was mostly maintained at broad regions of PRC2 occupancy such as those found at the Hox loci, the proteins were reduced at narrower regions of PRC2 occupancy demonstrating a particular requirement for the $\mathrm{ZnB} / \mathrm{Zn}$ region for $\mathrm{PRC} 2$ recruitment to these sites.

415

Measurement of H3K27me3 and H4Kac at PRC2 target sites revealed that JAZF1-SUZ12containing PRC2 is less catalytically active than WT SUZ12-containing PRC2; sites with comparable levels of JAZF1-SUZ12 and SUZ12 occupancy exhibit reduced H3K27me3 in cells expressing JAZF1-SUZ12. This was also the case for SUZ12 $\triangle 93$-containing PRC2, indicating that

420 the reduction of PRC2 catalytic activity in the presence of JAZF1-SUZ12 is due to loss of interaction with JARID2, EPOP and PALI1. This is consistent with the role of JARID2 (Sanulli et al., 2015) and PALI1 (Zhang et al., 2020) in the allosteric activation of EZH2. Due to this loss of catalytic activity, ectopic JAZF1-SUZ12 binding was not generally accompanied by ectopic regions of $\mathrm{H} 3 \mathrm{~K} 27 \mathrm{me} 3$.

Coupled with this decrease in H3K27me3, JAZF1-SUZ12 also increased H4Kac at PRC2 target sites. Increases in H4Kac at PRC2 target genes could also be observed in ESC expressing SUZ12 $\triangle 93$, indicating that they were primarily due to loss of repressive H3K27me3. However, in EBs, JAZF1-SUZ12 had a more marked effect than SUZ12 993 on H4Kac, indicating that this 430 reflected gain-of-function caused by recruitment of NuA4/TIP60 to these sites. Further work will 
be necessary to determine whether fusion of JAZF1 to SUZ12 also increases other NuA4-mediated chromatin modifications (H2A.Z incorporation, H2A/H2A.Z acetylation) at PRC2 target genes.

The changes in SUZ12 recruitment and histone modification caused by fusion to JAZF1 was

435 reflected in dysregulated patterns of gene expression and differentiation of cells expressing JAZF1-SUZ12. LG-ESS has previously been shown to exhibit elevated expression of polycomb target genes compared with leiomyosarcoma (Przybyl et al., 2018). Thus, our finding that JAZF1SUZ12 reduces H3K27me3 and increases H4Kac at PRC2 target genes provides an explanation for this elevated expression. Our results further suggest that these changes in H3K27me3 and

440 H4Kac disrupt normal programs of cell differentiation and that this contributes to the role of JAZF1-SUZ12 in oncogenesis. However, confirmation of this hypothesis will require the development of a cell or animal model in which JAZF1-SUZ12 promotes endometrial stromal cell tumourigenesis.

445 In summary, we have defined the changes in PRC2 composition, recruitment and activity caused by the JAZF1-SUZ12 fusion protein and the effects of these alterations on gene expression and cell differentiation. This work provides new insights into the regulation of PRC2 function and the role of JAZF1-SUZ12 in oncogenesis.

\section{AUTHOR CONTRIBUTIONS}

MT and RGJ designed experiments. MT and MB performed experiments. MT, GK and RGJ analysed data. KV and JM generated reagents. JB and RGJ supervised the research. MT and RGJ wrote the paper. 


\section{ACKNOWLEDGMENTS}

We thank the UCL Cancer Institute Genomics Core Facility and UCL Genomics for sequencing. We would like to thank Diego Pasini (Istituto Europeo di Oncologia), Luciano Di Croce (Centre for Genomic Regulation, Barcelona), Adrian Bracken (Trinity College Dublin), Neil Brockdorff (University of Oxford), Amanda Fisher (London Institute for Medical Sciences), Paola Scaffidi

460 (Francis Crick Institute) and Bart Vanhaesebroeck (UCL Cancer Institute) for cell lines and plasmids, and staff in the Implantation Research Clinic at University Hospitals Coventry and Warwickshire NHS Trust for endometrial sample collection. The research was funded by grants from the European Research Council (ERC, 311704) and Worldwide Cancer Research (13-0256) to RGJ, CoNaCyT (411064) to MT and a Wellcome Trust Investigator Award (212233/Z/18/Z) to

465 JJB. GK is a member of the Bill Lyons Informatics Centre and was funded by the Cancer Research UK UCL Centre (award C416/A18088).

\section{DECLARATION OF INTERESTS}

The authors declare no competing interests. 


\section{METHODS}

\section{Cell culture}

ESC lines were maintained on $0.1 \%$ gelatin coated dishes. E14, Suz $12^{\mathrm{GT} / \mathrm{GT}}$ (gift from Diego Pasini), $E z h^{\mathrm{fl} / \mathrm{fl}}, A e b p 2^{\mathrm{WT} / \mathrm{WT}}, A e b p 2^{\mathrm{GT} / \mathrm{GT}}$ (gifts from Neil Brockdorff) and Jarid2 ${ }^{\mathrm{GT} / \mathrm{GT}}$ (gift from Amanda Fisher) ESC were cultured in KO-DMEM (ThermoFisher, 10829018), 10\% FBS validated for mouse ESC culture (ThermoFisher, 16141079), 5\% knockout serum replacement

480 (ThermoFisher, 10828028), non-essential amino acids (ThermoFisher, 11140035), L-glutamine 2 $\mathrm{mM}$ (ThermoFisher, 25030-024), $50 \mu \mathrm{M}$ 2-mercaptoethanol (ThermoFisher, 31350010), 100 U/ml penicillin-streptomycin (ThermoFisher, 15140-122), 1 mM sodium pyruvate (ThermoFisher, 11360039) and $1000 \mathrm{U} / \mathrm{ml}$ leukemia inhibitory factor (Stemgent, 03-0011-100). Pali1 ${ }^{\mathrm{WT} / \mathrm{WT}}$, Pali1 $^{\mathrm{GT} / \mathrm{GT}}$ and $P c l 2^{\mathrm{GT} / \mathrm{GT}} P c l 1 / 3^{\mathrm{cKO}}$ (gifts from Adrian Bracken) ESC were maintained in GMEM

485 (Sigma, G5154) with the same supplements, except with no serum replacement and replacing Lglutamine with GlutaMAX (Thermofisher, 35050038), as were Epop ${ }^{\mathrm{GT} / \mathrm{GT}}$ and Epop ${ }^{\mathrm{WT} / \mathrm{WT}}$ cell lines (gifts from Luciano Di Croce) (Beringer et al., 2016) except for 20\% FBS. Deletion of Pcll and $P c l 3$ was induced by treating $P c l 2^{\mathrm{GT} / \mathrm{GT}} P c l 1 / 3^{\mathrm{cKO}}$ cells with $0.5 \mu \mathrm{M}$ 4-hydroxytamoxifen (Sigma, H6278) for 72 hrs (Healy et al., 2019). NIH-3T3 cells (gift from Bart Vanhaesebroeck) and Lenti-

490 X 293T (Takara Bio Europe) were cultured in high glucose DMEM (Thermofisher, 31966-047), 10\% FBS (Thermofisher, 10270-106) and penicillin-streptomycin. Immortalized primary human fibroblasts (gift from Paola Scaffidi) expressing human telomere reverse transcriptase (hTERT) (Scaffidi and Misteli, 2011) were grown in MEM (Thermofisher, 11095-080) supplemented with 15\% FBS, $100 \mathrm{U} / \mathrm{mL}$ penicillin streptomycin and $2 \mathrm{mM}$ L-glutamine. Drosophila S2 cells (gift 495 from Ivana Bjedov) were grown in Schneider's Drosophila Medium (ThermoFisher, Cat.no. 
21720-02) supplemented with 10\% heat-inactivated FBS (ThermoFisher, 10500064) and 25 U/mL of penicillin-streptomycin (ThermoFisher 15140-122). All cell lines were tested negative for mycoplasma (Lonza, LT07-701).

ESC were differentiated into EBs as described previously (Brien et al., 2012). ESC were filtered using a $70 \mu \mathrm{m}$ cell strainer, washed twice with LIF-free media and seeded as a single cell

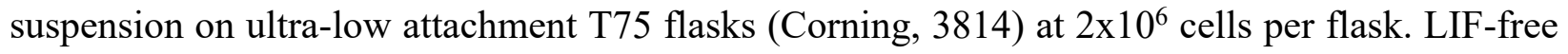
media was changed every second day and EBs were harvested at days 4 and 8 . For quantification

505 of EB growth, 200 cells per well were seeded in $200 \mu$ l of LIF-free media in a 96-well ultra-low attachment plate (Costar, 7007) and media was changed every third day. Pictures of single wells were taken with a EVOS FL Auto Imaging System at days 2, 4, 6 and 8, and EB diameter was measured by ImageJ using a macro that used the $650 \mu \mathrm{m}$ bar in each image as reference.

\section{Cloning}

Human SUZ12 and SUZ12 493 were PCR amplified from cDNA. JAZF1 was PCR amplified from IMAGE clone 4814463. To generate JAZF1-SUZ12, nucleotides encoding amino acids 1-128 of JAZF1 and amino acids 94-739 of SUZ12 were PCR amplified and joined in-frame using synonymous AccIII sites incorporated into the PCR primers. The constructs were cloned into 515 pCBA-HA and also into pCAG-GFP-2xFLAG (gift from Amanda Fisher). For tagging with Streptags, ORFs were PCR amplified from the pCAG plasmids (primers: 5'-TACTTCCAATCCATG and 5'-TATCCACCTTTACTG), C overhangs added with T4 DNA polymerase (M4211), and hybridised with BaeI-linearised pCAG-FS2-LIC to which G overhangs had been added. To 
generate retroviral vectors, ORFs were excised from pCAG-2xFLAG with 5'EcoRI and 3'NotI 520 and ligated into pMY-IRES-bls.

\begin{tabular}{|l|l|}
\hline \multicolumn{1}{|c|}{ Plasmid } & \multicolumn{1}{c|}{ Source } \\
\hline pCAG-GFP(FLAG) & A. Fisher \\
\hline pCAG-SUZ12(FLAG) & This work \\
\hline pCAG-SUZ12D93(FLAG) & This work \\
\hline pCAG-JAZF1-SUZ12(FLAG) & This work \\
\hline pCAG-JAZF1(FLAG) & This work \\
\hline pCAG-(FS2)GFP & This work \\
\hline pCAG-(FS2)SUZ12 & This work \\
\hline pCAG-(FS2)SUZ12D93 & This work \\
\hline pCAG-(FS2)JAZF1-SUZ12 & This work \\
\hline pCAG-(FS2)JAZF1 & This work \\
\hline pMY-IRES-bls-GFP(FLAG) & This work \\
\hline pMY-IRES-bls-SUZ12(FLAG) & This work \\
\hline pMY-IRES-bls-JAZF1-SUZ12(FLAG) & This work \\
\hline pMY-IRES-bls-JAZF1(FLAG) & This work \\
\hline pCBA-SUZ12 (HA) & This work \\
\hline pCBA-SUZ12D93 (HA) & This work \\
\hline pCBA-JAZF1-SUZ12 (HA) & This work \\
\hline pCBA-JAZF1 (HA) & This work \\
\hline pCAG-(FS2)AEBP2 & N. Brockdorff; Grijzenhout et al., 2016 \\
\hline pCAG-(FS2)EPOP & N. Brockdorff; Grijzenhout et al., 2016 \\
\hline pLenti-(FLAG/HA) PALI1 & A. Bracken; Healy et al., 2019 \\
\hline PCL3-V5 & J. Reiter; Hunkapiller et al., 2012 \\
\hline
\end{tabular}

\section{Transfection and generation of cell lines}

pCAG constructs were transfected into Suz12 GT/GT ESC with Effectene (Qiagene, 301425), following the manufacturer's protocol, cells selected with $2 \mu \mathrm{g} / \mathrm{ml}$ puromycin and stable cell lines

525 expanded from single cell colonies. NIH3T3 cells were transfected with $1 \mu \mathrm{g} / \mathrm{ml}$ plasmid with polyethyleneimine ( $3 \mu \mathrm{g} / \mathrm{ml}$; Polysciences, 23966). Media was changed the next day and cells harvested 48 hrs later. Retroviruses were produced by transfection of lenti-X 293T cells with 1.5 $\mu \mathrm{g}$ of pMY-IRES-bls constructs, $1 \mu \mathrm{g}$ of pCMVi and $1 \mu \mathrm{g}$ of pMDG (gifts from Greg Towers) 
using Fugene-HD (Promega, E2311). Virus was collected from 4x $10 \mathrm{~cm}$ plates 48, 72 and $96 \mathrm{hrs}$

530 after transfection and concentrated at $17,000 \times \mathrm{g}$ for $2 \mathrm{hrs}$ at $4^{\circ} \mathrm{C}$. The viral pellet was resuspended in $1 \mathrm{ml} \mathrm{FBS}$-free DMEM/F12 and then supplemented with 10\% DCC FBS prior to freezing at $80^{\circ} \mathrm{C}$. To make the FLAG-SUZ12 spike-in control material for ChIP-seq, hTERT cells were transduced with $1.5 \mathrm{ml}$ of concentrated pMY-SUZ12-FLAG-IRES-bls virus in the presence of 8 $\mu \mathrm{g} / \mathrm{ml}$ polybrene (Merck, TR-1003-G). Cells were then spinoculated at $500 \mathrm{x} \mathrm{g}$ for $1 \mathrm{hr}$ at RT and

535 incubated for 12 hrs. Cells were washed with fresh media and, after 3 days, selected with $5 \mu \mathrm{g} / \mathrm{ml}$ blasticidin.

\section{hEnSC cell culture, transduction and decidualisation}

The collection of endometrial biopsies was approved by the NHS National Research Ethics

540 - Hammersmith and Queen Charlotte's \& Chelsea Research Ethics Committee (REC reference: 1997/5065) and Tommy's National Reproductive Health Biobank (REC reference: 18/WA/0356). Samples were obtained using a Wallach Endocell sampler 5 to 10 days after the pre-ovulatory luteinizing hormone $(\mathrm{LH})$ surge. hEnSC were harvested as previously described (Barros, Brosens and Brighton, 2016). Briefly, endometrial biopsies were subjected to enzymatic 545 digestion using $500 \mu \mathrm{g} / \mathrm{ml}$ collagenase type Ia (Sigma-Aldrich,) and $100 \mu \mathrm{g} / \mathrm{mL}$ DNase I (Lorne Laboratories Ltd) for $1 \mathrm{hr}$ at $37^{\circ} \mathrm{C}$. Digested tissue was filtered through a $40 \mu \mathrm{m}$ cell strainer to remove glandular cell clumps, and the flow-through collected and cultured in DMEM/F12 (Thermo Scientific, 31330038) containing 10\% dextran-coated charcoal-treated foetal bovine serum (DCC-FBS), $1 \times$ antibiotic-antimycotic mix (ThermoFisher, 15240062), 10 $550 \mu \mathrm{M}$ L-glutamine, $1 \mathrm{nM}$ estradiol (Sigma, E2758) and $2 \mu \mathrm{g} / \mathrm{mL}$ insulin (Santa Cruz, sc-360248) . 
Cells were reseeded at a 1:2 or 1:3 ratio when confluence was reached. All experiments were carried out before reaching the $10^{\text {th }}$ passage.

$\mathrm{hEnSC}$ were seeded at a confluency of $10^{5}$ cells/well in 6 well plates and $1.5 \mathrm{ml}$ of concentrated

555 virus added to each well in the presence of $8 \mu \mathrm{g} / \mathrm{ml}$ polybrene (Merck, TR-1003-G). Cells were then spinoculated at $500 \mathrm{x}$ g for $1 \mathrm{hr}$ at RT and incubated for $12 \mathrm{hrs}$. Cells were washed with fresh media and, after 3 days, selected with $5 \mu \mathrm{g} / \mathrm{ml}$ blasticidin until they reached near confluency and no cells were found in the non-transduced control plate. Cells were reseeded and, at confluency two days later, decidualised for up to 8 days in DCC media with 2\% DCC FBS supplemented with 560 antibiotic antimycotic solution, l-glutamine, $50 \mu \mathrm{M}$ 8-Bromoadenosine 3', 5' cyclic monophosphate (8-Br-cAMP, Merck B5386) and $1 \mu \mathrm{M}$ medroxyprogesterone acetate (MPA, Sigma M1629), with the media changed every second day.

\section{Co-immunoprecipitation and Strep-Tactin pull-down}

565 Immunoprecipitations were performed as described (Conway et al., 2018), with minor modifications. 50 million cells per IP were suspended in $500 \mu$ l of high salt buffer (50 mM Tris$\mathrm{HCl}, \mathrm{pH}$ 7.2, $300 \mathrm{mM} \mathrm{NaCl}, 0.5 \%$ (v/v) NP-40, 1 mM EDTA pH7.4, Complete protease inhibitor (Roche, 11873580001) and $1 \mathrm{mM}$ DTT) and sonicated for 3x 10 seconds using a Bioruptor Pico (Diagenode). Cells were then rotated at $4^{\circ} \mathrm{C}$ for 20 mins before the lysates were diluted with 500 $570 \mu \mathrm{l}$ of no salt buffer $(50 \mathrm{mM}$ Tris-HCl, $\mathrm{pH} 7.2,0.5 \%(\mathrm{v} / \mathrm{v}) \mathrm{NP}-40,1 \mathrm{mM}$ EDTA pH7.4, Complete protease inhibitor and freshly added $1 \mathrm{mM}$ DTT). Afterwards, lysates were clarified by centrifugation for $10 \mathrm{~min}$ at $17,000 \mathrm{xg}$ at $4^{\circ} \mathrm{C}$ and $50 \mu \mathrm{l}$ taken from the sample for use as input. The remainder of the lysate was incubated with $2 \mu \mathrm{g}$ of anti-FLAG M2 (Sigma, F1804), SUZ12 
(CST, 3737) or anti-V5 (Abcam, ab15828) antibody with $250 \mathrm{U} / \mathrm{mL}$ benzonase (Sigma, E1014)

575 for 16 hrs. $50 \mu$ Protein G Dynabeads (Invitrogen, 10003D) per sample were washed 3 times with wash buffer (1:1 dilution of high salt: no salt buffer), resuspended in their initial volume, and incubated with the protein lysates for $2 \mathrm{hrs}$ with rotation at $4^{\circ} \mathrm{C}$. The flow-through was removed and immunocomplexed beads washed 5 times with $1 \mathrm{ml}$ of wash buffer. Beads were resuspended in $100 \mu \mathrm{l}$ of a 1:1 mix of $2 \mathrm{X}$ Laemmli buffer (4\% SDS, $240 \mathrm{mM}$ Tris $\mathrm{pH} 6.7$, 2\% beta-

580 mercaptoethanol, $20 \%$ glycerol and $0.2 \%$ bromophenol blue) and $10 \mathrm{mM}$ Tris HCl, while $50 \mu 1$ of 2X Laemmli buffer was added to input samples and both heated at $95^{\circ} \mathrm{C}$ for $5 \mathrm{~min}$. Finally, $10 \mu \mathrm{l}$ of each IP and input sample were resolved by SDS-PAGE.

Strep-Tactin pull-down was carried out as for FLAG immunoprecipitation, with the following 585 modifications. To block binding of any unspecific biotinylated proteins, $10 \mu \mathrm{g} / \mathrm{ml}$ of avidin (IBA, Cat No. 2-0204-015) was added to the extracts after cell lysis. Cell extracts were then incubated for $30 \mathrm{~min}$ at $4^{\circ} \mathrm{C}$ with rotation and centrifuged at $20,817 \mathrm{x}$ g for $5 \mathrm{~min}$ at $4^{\circ} \mathrm{C}$ and the supernatant recovered. Supernatant was added to $10 \mu \mathrm{l}$ of StrepTactin superflow high-capacity resin (IBA, Cat no. 2-1208-002) and incubated with rotation for $4 \mathrm{hrs}$ at $4^{\circ} \mathrm{C}$. Resin was then washed 5 times with

590 wash buffer, each time pelleting the resin at $1000 \mathrm{x}$ g for 5 mins at $4^{\circ} \mathrm{C}$. Protein was eluted by boiling the resin in Laemmli buffer.

\section{Nuclear fractionation and RNaseA treatment}

RNaseA treatment and cell fractionation was performed as described (Beltran et al., 2016; Zoabi

595 et al., 2014). ESCs were trypsinized, washed twice with PBS, permeabilized with $0.05 \%$ Tween20 in PBS for 10 mins on ice, washed once, resuspended with PBS and mock-treated or treated 
with $1 \mathrm{mg} / \mathrm{ml}$ RNaseA (Sigma, R6513) for 30 mins at RT. Cells were centrifuged at $1200 \mathrm{rpm}$, washed twice and resuspended in $1 \mathrm{ml}$ of buffer A (10 mM HEPES (pH 7.9), $10 \mathrm{mM} \mathrm{KCl,} 1.5 \mathrm{mM}$ MgC12, $0.34 \mathrm{M}$ sucrose, 10\% glycerol, $1 \mathrm{mM}$ DTT with Complete protease inhibitor). Triton X-

$600100(0.1 \%)$ was added, and the cells were incubated for 5 mins on ice. Nuclei were collected by low-speed centrifugation ( $4 \mathrm{mins}, 1,300 \mathrm{x} \mathrm{g}, 4^{\circ} \mathrm{C}$ ). The supernatant (cytoplasmic fraction) was further clarified by high-speed centrifugation $\left(15 \mathrm{~min}, 20,000 \mathrm{x} \mathrm{g}, 4^{\circ} \mathrm{C}\right)$. Nuclei were washed twice in buffer A, and then lysed in buffer B (3 mM EDTA, $0.2 \mathrm{mM}$ EGTA, $1 \mathrm{mM}$ DTT, Complete protease inhibitor). Insoluble chromatin was collected by centrifugation $\left(4 \mathrm{~min}, 1,700 \mathrm{x} \mathrm{g}, 4^{\circ} \mathrm{C}\right)$,

605 and the supernatant (nucleoplasmic fraction) was recovered. The final chromatin pellet (chromatin fraction) was washed twice with buffer $\mathrm{B}$, resuspended in $1 \mathrm{X}$ Laemmli buffer, sonicated (Diagenode Bioruptor Pico) and resolved by SDS-PAGE.

\section{Immunoblotting}

610 Proteins were resolved along side PageRuler (ThermoFisher, 26620) by SDS-PAGE using the Mini-PROTREAN Tetra Cell system (BioRad) in $200 \mathrm{mM}$ glycine, $24 \mathrm{mM}$ Tris base and 0.1\% SDS. Proteins were transferred to $0.45 \mu \mathrm{M}$ nitrocellulose membrane (GE Healthcare, 15269794) using a Mini Trans-Blot system (Biorad, 1610158) at $350 \mathrm{~mA}$ for 2 hrs. Membranes were blocked with 5\% non-fat dried milk plus $0.1 \%$ Tween (Sigma, P1379) in TBS (TBST) for $1 \mathrm{hr}$ at RT.

615 Proteins were detected with primary antibodies to FLAG M2 (Sigma, A8592), HA 3F10 (Roche, 12013819001), V5 (Abcam, ab15828), SUZ12 (Santa Cruz sc-46264), EZH2 (CST, 3147), JARID2 (CST, 13594), AEBP2 (CST 14129), PCL2 (Proteintech 16208-1-AP), EPOP (kind gift of L. Di Croce), TRRAP (Abcam, ab73546), FUS (Novus Biologicals 100-565), $\beta$-actin (CST 4967), alpha tubulin (CST 2144), H3K27me3 (Abcam ab192985) and H3 (Abcam ab1791) and 
620 HRP-conjugated secondary antibodies (anti-mouse (Dako, P0447) or anti-rabbit (Dako, P0448)).

Proteins were visualised using the Clarity ECL Western Substrate (Biorad, 1705061) and detected using an ImageQuantLAS 4000 imager and ImageQuantTL software (GE). Contrast and brightness were altered in a linear fashion equally across the whole image.

\section{RNA quantification}

RNA was purified using TRIsure (Bioline, BIO-38033) and reverse transcribed using the ImProm-II Reverse Transcription System (Promega, A3800) and random hexamer primers. Specific RNAs were quantified using the QuantiTect SYBR Green PCR Kit (Qiagen, 204145) and a QuantStudio 5 Real-Time PCR System (Thermofisher) with the primers shown below.

\begin{tabular}{|c|c|c|c|}
\hline Species & Gene & Forward 5'-3' & Reverse $5^{\prime}-3^{\prime}$ \\
\hline \multirow{10}{*}{ Mouse } & Nanog & CCTCCAGCAGATGCAAGAACTC & CTTCAACCACTGGTTTTTCTGCC \\
\hline & Pou5f1 (Oct4) & GCTCACCCTGGGCGTTCTC & GGCCGCAGCTTACACATGTTC \\
\hline & Klf4 & GTTTTTAATCTTCGTTGACTTTGGG & CAGCCATGTCAGACTCGCC \\
\hline & $\mathrm{Fgf4}$ & CTACTGCAACGTGGGCATCG & CGCTGCACCGGAGAGAGC \\
\hline & Utfl & ACCAGATCCGCCAACTCATGGG & TCGTCGTGGAAGAACTGAA \\
\hline & $F g f 5$ & CTGCAAGATGCACTTAGGACCC & TGAGGAAGAGCAAGGACAGGC \\
\hline & $\operatorname{Pax} 3$ & TCCCATGGTTGCGTCTCTAAG & CTCCACGTCAGGCGTTGTC \\
\hline & $\operatorname{Brachyury}(T)$ & TTTCTTGCTGGACTTCGTGACG & CCACTCCCCGTTCACATATTTC \\
\hline & Gata4 & CACAAGATGAACGGCATCAACC & CAGCGTGGTGGTGGTAGTCTG \\
\hline & Gapdh & ATGATGCGCAAAGGTATGCA & ССССАТСТСССССТТССТ \\
\hline \multirow{4}{*}{ Human } & $P R L$ & TGCTTTAACATTTTTGCCTAGTAA & AAATGGAGTGTCTAA AAACGTTGA \\
\hline & $I G F B P 2$ & CACTTGTGAGAAGCGCCG & GGTCATCGCCATTGTCTGC \\
\hline & ROR2 & GTCCAACGCACAGCCCAAATC & CCGGTTGCCAATGAAGCGTG \\
\hline & 5s rRNA & GATCTCGGAAGCTAAGCAGG & AAGCCTACAGCACCCGGTAT \\
\hline \multirow{3}{*}{ Transgenes } & $\begin{array}{l}\text { SUZ12 } \\
\text { constructs }\end{array}$ & AAGGCAGTAACTAAGCTCCGTG & CGTTTGCAGGGGAAGCAGAT \\
\hline & $\begin{array}{l}\text { JAZF1- } \\
\text { SUZ12 } \\
\end{array}$ & TTCATTCCGGAGCAGCACTC & TGGTGCTATGAGATTCCGAGTT \\
\hline & JAZF1-FLAG & CAGATTCGTGTCCGCAAACC & TGTAATCGGTACCGCTAGCTT \\
\hline
\end{tabular}




\section{Calibrated ChIP-sequencing (cChIP-Seq)}

All ChIP-seq experiments were performed in duplicate. Cells were trypsinised, resuspended in PBS and then crosslinked by adding $1 / 10$ volume of cross-linking solution ( $11 \%$ formaldehyde,

$6350.1 \mathrm{M} \mathrm{NaCl}, 1 \mathrm{mM}$ EDTA $\mathrm{pH}$ 8, $0.5 \mathrm{mM}$ EGTA pH 8, $50 \mathrm{mM}$ HEPES pH 8) for $15 \mathrm{~min}$. Formaldehyde was quenched with $1.25 \mathrm{mM}$ glycine. Cells were washed twice with ice-cold PBS, centrifuging at $290 \mathrm{x} \mathrm{g}$ at $4^{\circ} \mathrm{C}$ for $10 \mathrm{~min}$ each time, flash-frozen and stored at $-80^{\circ} \mathrm{C}$. Cells were thawed, resuspended in lysis buffer 1 (50 mM HEPES pH 7.5, $140 \mathrm{mM} \mathrm{NaCl}, 1 \mathrm{mM}$ EDTA, $10 \%$ glycerol, $0.5 \%$ IGEPAL CA-630, $0.25 \%$ Triton X-100) and incubated at $4^{\circ} \mathrm{C}$ for 10 mins with

640 rocking. Cells were recovered by centrifugation at $290 \mathrm{x}$ g for $10 \mathrm{~min}$ at $4^{\circ} \mathrm{C}$ and nuclei resuspended in the same volume of lysis buffer 2 (10 mM Tris $\mathrm{pH} 8,200 \mathrm{mM} \mathrm{NaCl}, 1 \mathrm{mM}$ EDTA, $0.5 \mathrm{mM}$ EGTA, supplemented with 0.1 DTT and Complete protease inhibitor) and incubated at $4^{\circ} \mathrm{C}$ for 10 mins with rocking. Nuclei were pelleted again at $290 \mathrm{x}$ g for $10 \mathrm{mins}$ at $4^{\circ} \mathrm{C}$ and resuspended in $100 \mu \mathrm{l}$ of lysis buffer $3(10 \mathrm{mM}$ Tris $\mathrm{pH} 8,100 \mathrm{mM} \mathrm{NaCl}, 1 \mathrm{mM}$ EDTA, 0.5mM

645 EGTA, 0.1\% sodium deoxycholate, 0.5\% N-lauryl sarcosine, $0.2 \%$ SDS, supplemented with 0.1 DTT and Complete protease inhibitor) per $5 \times 10^{6}$ cells. Cells were incubated for 30 mins on ice and sonicated for 15 cycles of 30s on and 30s off using a Diagenode Bioruptor Pico. 2x107 ESC or the EBs formed from 2x $10 \mathrm{~cm}$ plates (4 day timepoint) or $1 \times 10 \mathrm{~cm}$ plate (8 day timepoint) were used per ChIP. An aliquot of the sonicated chromatin was reverse crosslinked by heating at $65065^{\circ} \mathrm{C}$ for $1 \mathrm{hr}$, treated with $0.5 \mathrm{mg} / \mathrm{ml} \mathrm{RNaseA}$ and $0.2 \mathrm{mg} / \mathrm{ml}$ of proteinase $\mathrm{K}$ (Ambion, AM2546).

DNA was purified from this aliquot using KAPA Pure beads (Roche, KK8000), resuspended in $10 \mathrm{mM}$ Tris $\mathrm{pH} 8$ and quantified using the Qubit dsDNA HS Assay kit (Thermofisher, Q32851). Each ChIP was then performed with mouse chromatin equivalent to $45 \mu \mathrm{g}$ of purified DNA. For ChIPs for FLAG-tagged proteins, sonicated human chromatin (extracted from hTERT cells stably 
655 expressing FLAG-SUZ12) equivalent to $5 \mu \mathrm{g}$ of purified DNA was added as a calibration control. For ChIPs for histone modifications, Drosophila chromatin (extracted from S2 cells) equivalent to $5 \mu \mathrm{g}$ of purified DNA was added. The lysates were topped up to $900 \mu \mathrm{l}$ per IP, $100 \mu \mathrm{l}$ Triton X$10010 \%$ solution added, and insoluble material removed by centrifugation at 17,000 x g for 20 mins at $4{ }^{\circ} \mathrm{C} .2 \%$ of the lysate was stored at $-20^{\circ} \mathrm{C}$ to be used as input and the remaining lysate was

660 incubated overnight at $4^{\circ} \mathrm{C}$ with $50 \mu$ protein $\mathrm{G}$ Dynabeads preincubated for at least $4 \mathrm{hr}$ with 2.5 $\mu \mathrm{g}$ of anti-FLAG M2 (Sigma, A8592), H3K27me3 (Abcam, ab192985) or H4 pan-acetyl (Thermofisher, PA5-40083) antibody. Beads were washed six times with wash buffer (50 mM Hepes, $1 \mathrm{mM}$ EDTA, $0.5 \mathrm{M} \mathrm{LiCl,}$ 0.7\% sodium deoxycholate, 1\% NP-40) and once with TE with $50 \mathrm{mM} \mathrm{NaCl}$ and bound complexes eluted in elution buffer (50 mM Tris, $10 \mathrm{mM}$ EDTA, 1\% SDS).

665 Crosslinks were reversed by heating at $65^{\circ} \mathrm{C}$ for $8 \mathrm{hrs}$ and DNA purified as before.

Sequencing libraries were generated from $1.2 \mathrm{ng}$ of DNA using the NEBNext Ultra II DNA library preparation kit (NEB, E7645) with 9 cycles of PCR. Library quality and size distribution was assessed using the 2100 Bioanalyzer High Sensitivity DNA Kit (Agilent, 5067-4626) followed by

670 qPCR quantification with the Kapa Library Quant Kit (Roche, KK4824). FLAG libraries were subjected to $75 \mathrm{bp}$ single-end sequencing on an Illumina NextSeq 550 platform and histone modification libraries were subjected to $138 \mathrm{bp}$ single-end sequencing on an Illumina NovaSeq platform.

\section{ChIP-seq data analysis}

GFP, SUZ12, SUZ12 $\triangle 93$, JAZF1-SUZ12 and JAZF1 ChIP-seq reads were aligned to a concatenated genome sequence of mouse (mm10) and human (hg19) using bowtie2 with the -- 
very-sensitive option (Langmead and Salzberg, 2012). Uniquely mapped reads were extracted using samtools (Li et al., 2009) and were used for the downstream analysis. For each sample, 680 endogenous (mouse) and exogenous (human) reads were segregated into two bam files using samtools.

Spike-in calibration was performed as previously described (Fursova et al., 2019) and downsampled bam files were generated for the endogenous mouse data. The two replicates for the 685 endogenous data were merged and peaks were called using MACS2 with the --broad option and -broad-cutoff $=0.0001$ (Feng et al., 2012). Peaks overlapping mouse blacklist regions (Amemiya et al., 2019) or peaks called in any of the GFP control datasets were removed using bedTools (Quinlan and Hall, 2010). Genome coverage tracks were obtained using MACS2 pileup function, which were then converted to bigwigs and visualized with the UCSC Genome Browser (Kent et 690 al., 2002).

A combined peak-set was created from the merged peaks from all the factors at the three timepoints using DiffBind in R with the default parameters (Ross-Innes et al., 2012). Correlations between the peaks in different samples were calculated with DiffBind using the Pearson correlation

695 co-efficient. Peak overlaps were calculated with dba.overlap and UpSet plots generated with UpSetR (Conway et al., 2017). Metaplots and heatmaps were generated with computeMatrix and plotProfile/plotHeatmap functions from deepTools (Ramírez et al., 2014).

H3K27me3 and H4Kac ChIP-seq reads were aligned to a concatenated genome sequence of mouse 700 (mm10) and Drosophila (BDGP5.25) using bowtie2 with the --very-sensitive option. Uniquely 
mapped reads were extracted and separated into endogenous (mouse) and exogenous (Drosophila) reads using samtools. The normalization factor for the mouse bams was calculated using the Drosophila spike-in according as previously described (Orlando et al., 2014). The normalization factor was used to generate the mouse bigwigs using the bamCoverage function from deepTools.

The closest gene transcription start sites (TSS; Ensembl v98) to each SUZ12, JAZF1-SUZ12 and JAZF1 MACS peak was identified with bedTools. Gene TSS within $1 \mathrm{~kb}$ of a peak were considered to be bound. Gene Ontology terms enriched in the sets of genes bound by each factor were identified using g:Profiler with the default settings (Raudvere et al., 2019).

\section{Statistical analysis}

Measurements of chromatin association after RNA degradation were performed in triplicate and mean and standard deviation plotted in GraphPad Prism (GraphPad Software, USA) The significance of changes in chromatin association were estimated using a two-tailed Student's t-

715 test. Measurements of relative gene expression during EB formation were performed in quadruplicate, mean and standard deviation plotted and the significance of differences between samples were estimated using 2-way ANOVA using GraphPad Prism. Mean and standard deviation of EB size was plotted and the significance of differences between samples estimated using 2-way ANOVA (E14 n=95, GFP n=86, SUZ12 n=86, SUZ12 493 n=96, JAZF1-SUZ12

$720 \mathrm{n}=55$. Measurements of relative gene expression in $\mathrm{hEnSC}$ were performed in duplicate (cells from two different donors) and the significance of differences between samples were estimated using Student's t-test. 


\section{SUPPLEMENTAL INFORMATION}

$725 \quad$ Figures S1-S5

Table S1

\section{REFERENCES}

730 Alekseyenko, A. A., Gorchakov, A. a, Kharchenko, P. V, and Kuroda, M.I. (2014). Reciprocal interactions of human C10orf12 and C17orf96 with PRC2 revealed by BioTAP-XL cross-linking and affinity purification. Proc Natl Acad Sci USA 111, 2488-2493.

Amant, F., De Knijf, A., Van Calster, B., Leunen, K., Neven, P., Berteloot, P., Vergote, I., Van 735 Huffel, S., and Moerman, P. (2007). Clinical study investigating the role of lymphadenectomy, surgical castration and adjuvant hormonal treatment in endometrial stromal sarcoma. Br. J. Cancer 97, 1194-1199.

Amemiya, H.M., Kundaje, A., and Boyle, A.P. (2019). The ENCODE Blacklist: Identification of 740 Problematic Regions of the Genome. Sci. Rep. 9, 1-5.

Azuara, V., Perry, P., Sauer, S., Spivakov, M., Jørgensen, H.F., John, R.M., Gouti, M., Casanova, M., Warnes, G., Merkenschlager, M., et al. (2006). Chromatin signatures of pluripotent cell lines. Nat. Cell Biol. 8, 532-538.

Beck, T.L., Singhal, P.K., Ehrenberg, H.M., Rose, P.G., Lele, S.B., Krivak, T.C., and McBee, W.C. (2012). Endometrial stromal sarcoma: Analysis of recurrence following adjuvant treatment. Gynecol. Oncol. 125, 141-144.

750 Beltran, M., Yates, C.M., Skalska, L., Dawson, M., Reis, F.P., Viiri, K., Fisher, C.L., Sibley, C.R., Foster, B.M., Bartke, T., et al. (2016). The interaction of PRC2 with RNA or chromatin is mutually antagonistic. Genome Res. 26, 896-907.

Beltran, M., Tavares, M., Justin, N., Khandelwal, G., Ambrose, J., Foster, B.M., Worlock, K.B.,

755 Tvardovskiy, A., Kunzelmann, S., Herrero, J., et al. (2019). G-tract RNA removes Polycomb repressive complex 2 from genes. Nat. Struct. Mol. Biol. 26, 899-909.

Beringer, M., Pisano, P., Di Carlo, V., Blanco, E., Chammas, P., Vizán, P., Gutiérrez, A., Aranda, S., Payer, B., Wierer, M., et al. (2016). EPOP Functionally Links Elongin and Polycomb in 760 Pluripotent Stem Cells. Mol. Cell 64, 645-658.

Boyer, L.A., Plath, K., Zeitlinger, J., Brambrink, T., Medeiros, L.A., Lee, T.I., Levine, S.S., Wernig, M., Tajonar, A., Ray, M.K., et al. (2006). Polycomb complexes repress developmental regulators in murine embryonic stem cells. Nature 441, 349-353. 
Bracken, A.P., Dietrich, N., Pasini, D., Hansen, K.H., and Helin, K. (2006). Genome-wide mapping of polycomb target genes unravels their roles in cell fate transitions. Genes Dev. 20, 1123-1136.

770 Brien, G.L., Gambero, G., O’Connell, D.J., Jerman, E., Turner, S.A., Egan, C.M., Dunne, E.J., Jurgens, M.C., Wynne, K., Piao, L., et al. (2012). Polycomb PHF19 binds H3K36me3 and recruits PRC2 and demethylase NO66 to embryonic stem cell genes during differentiation. Nat. Struct. Mol. Biol. 19, 1273-1281.

775 Chen, S., Jiao, L., Shubbar, M., Yang, X., and Liu, X. (2018). Unique Structural Platforms of Suz12 Dictate Distinct Classes of PRC2 for Chromatin Binding. Mol. Cell 69, 840-852.e5.

Chiang, S., Ali, R., Melnyk, N., McAlpine, J.N., Huntsman, D.G., Gilks, C.B., Lee, C.H., and Oliva, E. (2011). Frequency of known gene rearrangements in endometrial stromal tumors. Am. J.

780 Surg. Pathol. 35, 1364-1372.

Comet, I., Riising, E.M., Leblanc, B., and Helin, K. (2016). Maintaining cell identity: PRC2mediated regulation of transcription and cancer. Nat. Rev. Cancer 16, 803-810.

785 Conway, J.R., Lex, A., and Gehlenborg, N. UpSetR: An R Package for the Visualization of Intersecting Sets and their Properties. (2017). Bioinformatics 33, 2938-2940.

Conway, E., Jerman, E., Healy, E., Ito, S., Holoch, D., Oliviero, G., Deevy, O., Glancy, E., Fitzpatrick, D.J., Mucha, M., et al. (2018). A Family of Vertebrate-Specific Polycombs Encoded 790 by the LCOR/LCORL Genes Balance PRC2 Subtype Activities. Mol. Cell 70, 408-421.e8.

Cuppens, T., Tuyaerts, S., and Amant, F. (2015). Potential Therapeutic Targets in Uterine Sarcomas. Sarcoma 2015, 1-14.

795 Davidovich, C., Zheng, L., Goodrich, K.J., and Cech, T.R. (2013). Promiscuous RNA binding by Polycomb repressive complex 2. Nat. Struct. Mol. Biol. 20, 1250-1257.

Davidson, B., and Micci, F. (2017). Molecular characteristics of uterine sarcomas. Expert Rev. Mol. Diagn. 17, 515-522.

Deevy, O., and Bracken, A.P. (2019). PRC2 functions in development and congenital disorders. Development 146, dev181354.

Dewaele, B., Przybyl, J., Quattrone, A., Finalet Ferreiro, J., Vanspauwen, V., Geerdens, E., 805 Gianfelici, V., Kalender, Z., Wozniak, A., Moerman, P., et al. (2014). Identification of a novel, recurrent MBTD1-CXorf67 fusion in low-grade endometrial stromal sarcoma. Int. J. Cancer 134, $1112-1122$.

Faust, C., Schumacher, A., Holdener, B., and Magnuson, T. (1995). The eed mutation disrupts 
Feng, J., Liu, T., Qin, B., Zhang, Y., and Liu, X.S. (2012). Identifying ChIP-seq enrichment using MACS. Nat. Protoc. 7, 1728-1740.

815 Fujikura, J., Yamato, E., Yonemura, S., Hosoda, K., Masui, S., Nakao, K., Miyazaki, J.I., and Niwa, H. (2002). Differentiation of embryonic stem cells is induced by GATA factors. Genes Dev. $16,784-789$.

Fursova, N.A., Blackledge, N.P., Nakayama, M., Ito, S., Koseki, Y., Farcas, A.M., King, H.W., 820 Koseki, H., and Klose, R.J. (2019). Synergy between Variant PRC1 Complexes Defines Polycomb-Mediated Gene Repression. Mol. Cell 74, 1020-1036.e8.

Garland, W., Comet, I., Wu, M., Radzisheuskaya, A., Rib, L., Vitting-Seerup, K., Lloret-Llinares, M., Sandelin, A., Helin, K., and Jensen, T.H. (2019). A Functional Link between Nuclear RNA 825 Decay and Transcriptional Control Mediated by the Polycomb Repressive Complex 2. Cell Rep. 29, 1800-1811.e6.

Gellersen, B., and Brosens, J.J. (2014). Cyclic decidualization of the human endometrium in reproductive health and failure. Endocr. Rev. 35, 851-905.

Grijzenhout, A., Godwin, J., Koseki, H., Gdula, M., Szumska, D., McGouran, J.F., Bhattacharya, S., Kessler, B.M., Brockdorff, N., and Cooper, S. (2016). Functional analysis of AEBP2, a PRC2 Polycomb protein, reveals a Trithorax phenotype in embryonic development and in ES cells. Development 2716-2723.

Han, L., Liu, Y.J., Ricciotti, R.W., and Mantilla, J.G. (2020). A novel MBTD1-PHF1 gene fusion in endometrial stromal sarcoma: A case report and literature review. Genes Chromosom. Cancer $428-432$.

840 Hauri, S., Comoglio, F., Seimiya, M., Gerstung, M., Glatter, T., Hansen, K., Aebersold, R., Paro, R., Gstaiger, M., and Beisel, C. (2016). A High-Density Map for Navigating the Human Polycomb Complexome. Cell Rep. 17, 583-595.

Healy, E., Mucha, M., Glancy, E., Fitzpatrick, D.J., Conway, E., Neikes, H.K., Monger, C., Van 845 Mierlo, G., Baltissen, M.P., Koseki, Y., et al. (2019). PRC2.1 and PRC2.2 Synergize to Coordinate H3K27 Trimethylation. Mol. Cell 76, 437-452.e6.

Hendrickson, D., Kelley, D.R., Tenen, D., Bernstein, B., and Rinn, J.L. (2016). Widespread RNA binding by chromatin-associated proteins. Genome Biol. 17, 1-18.

Højfeldt, J.W., Laugesen, A., Willumsen, B.M., Damhofer, H., Hedehus, L., Tvardovskiy, A., Mohammad, F., Jensen, O.N., and Helin, K. (2018). Accurate H3K27 methylation can be established de novo by SUZ12-directed PRC2. Nat. Struct. Mol. Biol. 25, 225-232.

855 Højfeldt, J.W., Hedehus, L., Laugesen, A., Tatar, T., Wiehle, L., and Helin, K. (2019). Non-core Subunits of the PRC2 Complex Are Collectively Required for Its Target-Site Specificity. Mol. 
Cell 76, 423-436.e3.

Hosogane, M., Funayama, R., Shirota, M., and Nakayama, K. (2016). Lack of Transcription Triggers H3K27me3 Accumulation in the Gene Body. Cell Rep. 16, 696-706.

Hübner, J.M., Müller, T., Papageorgiou, D.N., Mauermann, M., Krijgsveld, J., Russell, R.B., Ellison, D.W., Pfister, S.M., Pajtler, K.W., and Kool, M. (2019). EZHIP/CXorf67 mimics K27M mutated oncohistones and functions as an intrinsic inhibitor of PRC2 function in aggressive 865 posterior fossa ependymoma. Neuro. Oncol. 21, 878-889.

Jain, S.U., Do, T.J., Lund, P.J., Rashoff, A.Q., Diehl, K.L., Cieslik, M., Bajic, A., Juretic, N., Deshmukh, S., Venneti, S., et al. (2019). PFA ependymoma-associated protein EZHIP inhibits PRC2 activity through a H3 K27M-like mechanism. Nat. Commun. 10, 2146.

Kaneko, S., Son, J., Shen, S.S., Reinberg, D., and Bonasio, R. (2013). PRC2 binds active promoters and contacts nascent RNAs in embryonic stem cells. Nat Struct Mol Biol 20, 1258-1264.

Kaneko, S., Son, J., Bonasio, R., Shen, S.S., and Reinberg, D. (2014). Nascent RNA interaction 875 keeps PRC2 activity poised and in check. Genes Dev. 28, 1983-1988.

Kasinath, V., Faini, M., Poepsel, S., Reif, D., Feng, X.A., Stjepanovic, G., Aebersold, R., and Nogales, E. (2018). Structures of human PRC2 with its cofactors AEBP2 and JARID2. Science $359,940-944$.

Kent, W.J., Sugnet, C.W., Furey, T.S., Roskin, K.M., Pringle, T.H., Zahler, A.M., and Haussler, a. D. (2002). The Human Genome Browser at UCSC. Genome Res. 12, 996-1006.

Kloet, S.L., Makowski, M.M., Baymaz, H.I., van Voorthuijsen, L., Karemaker, I.D., Santanach,

A., Jansen, P.W.T.C., Di Croce, L., and Vermeulen, M. (2016). The dynamic interactome and genomic targets of Polycomb complexes during stem-cell differentiation. Nat. Struct. Mol. Biol. $23,682-690$.

Kobiita, A., Godbersen, S., Araldi, E., Ghoshdastider, U., Schmid, M.W., Spinas, G., Moch, H., 890 and Stoffel, M. (2020). The Diabetes Gene JAZF1 Is Essential for the Homeostatic Control of Ribosome Biogenesis and Function in Metabolic Stress. Cell Rep. 32, 107846.

Koontz, J.I., Soreng, a L., Nucci, M., Kuo, F.C., Pauwels, P., van Den Berghe, H., Dal Cin, P., Fletcher, J. A, and Sklar, J. (2001). Frequent fusion of the JAZF1 and JJAZ1 genes in endometrial 895 stromal tumors. Proc. Natl. Acad. Sci. U. S. A. 98, 6348-6353.

Kulinski, T.M., Casari, M.R.T., Guenzl, P.M., Wenzel, D., Andergassen, D., Hladik, A., Datlinger, P., Farlik, M., Theussl, H.C., Penninger, J.M., et al. (2015). Imprinted expression in cystic embryoid bodies shows an embryonic and not an extra-embryonic pattern. Dev. Biol. 402, 291900305.

Landeira, D., Sauer, S., Poot, R., Dvorkina, M., Mazzarella, L., Jørgensen, H.F., Pereira, C.F., 
Leleu, M., Piccolo, F.M., Spivakov, M., et al. (2010). Jarid2 is a PRC2 component in embryonic stem cells required for multi-lineage differentiation and recruitment of PRC1 and RNA 905 Polymerase II to developmental regulators. Nat. Cell Biol. 12, 618-624.

Langmead, B., and Salzberg, S.L. (2012). Fast gapped-read alignment with Bowtie 2. Nat. Methods 9, 357-359.

910 Lee, T.I., Jenner, R.G., Boyer, L.A., Guenther, M.G., Levine, S.S., Kumar, R.M., Chevalier, B., Johnstone, S.E., Cole, M.F., Isono, K., et al. (2006). Control of Developmental Regulators by Polycomb in Human Embryonic Stem Cells. Cell 125, 301-313.

Li, G., Margueron, R., Ku, M., Chambon, P., Bernstein, B.E., and Reinberg, D. (2010). Jarid2 and 915 PRC2, partners in regulating gene expression. Genes Dev. 24, 368-380.

Li, H., Ma, X., Wang, J., Koontz, J., Nucci, M., and Sklar, J. (2007). Effects of rearrangement and allelic exclusion of JJAZ1/SUZ12 on cell proliferation and survival. Proc. Natl. Acad. Sci. U. S. A. 104, 20001-20006.

920

Li, H., Handsaker, B., Wysoker, A., Fennell, T., Ruan, J., Homer, N., Marth, G., Abecasis, G., and Durbin, R. (2009). The Sequence Alignment/Map format and SAMtools. Bioinformatics 25, 20782079.

925 Li, H., Liefke, R., Jiang, J., Kurland, J.V., Tian, W., Deng, P., Zhang, W., He, Q., Patel, D.J., Bulyk, M.L., et al. (2017). Polycomb-like proteins link the PRC2 complex to CpG islands. Nature 549, 287-291.

Liefke, R., Karwacki-Neisius, V., and Shi, Y. (2016). EPOP Interacts with Elongin BC and USP7 930 to Modulate the Chromatin Landscape. Mol. Cell 64, 659-672.

Long, Y., Bolanos, B., Gong, L., Liu, W., Goodrich, K.J., Yang, X., Chen, S., Gooding, A.R., Maegley, K.A., Gajiwala, K.S., et al. (2017). Conserved RNA-binding specificity of polycomb repressive complex 2 is achieved by dispersed amino acid patches in EZH2. Elife 6, 1-23.

Ma, X., Wang, J., Wang, J., Ma, C.X., Gao, X., Patriub, V., and Sklar, J.L. (2016). The JAZF1/SUZ12 fusion protein disrupts PRC2 complexes and impairs chromatin repression during human endometrial stromal tumorogenesis. Oncotarget 8, 4062-4078.

940 Makise, N., Sekimizu, M., Kobayashi, E., Yoshida, H., Fukayama, M., Kato, T., Kawai, A., Ichikawa, H., and Yoshida, A. (2019). Low-grade endometrial stromal sarcoma with a novel MEAF6-SUZ12 fusion. Virchows Arch. 4-8.

Margueron, R., and Reinberg, D. (2011). The Polycomb complex PRC2 and its mark in life. Nature $945469,343-349$.

Micci, F., Panagopoulos, I., Bjerkehagen, B., and Heim, S. (2006). Consistent rearrangement of chromosomal band 6p21 with generation of fusion genes JAZF1/PHF1 and EPC1/PHF1 in 
endometrial stromal sarcoma. Cancer Res. 66, 107-112.

Micci, F., Gorunova, L., Agostini, A., Johannessen, L.E., Brunetti, M., Davidson, B., Heim, S., and Panagopoulos, I. (2016). Cytogenetic and molecular profile of endometrial stromal sarcoma. Genes, Chromosom. Cancer 55, 834-846.

955 Nakajima, T., Fujino, S., Nakanishi, G., Kim, Y.S., and Jetten, A.M. (2004). TIP27: A novel repressor of the nuclear orphan receptor TAK1/TR4. Nucleic Acids Res. 32, 4194-4204.

Nishibuchi, I., Suzuki, H., Kinomura, A., Sun, J., Liu, N.A., Horikoshi, Y., Shima, H., Kusakabe, M., Harata, M., Fukagawa, T., et al. (2014). Reorganization of damaged chromatin by the 960 exchange of histone variant H2A.Z-2. Int. J. Radiat. Oncol. Biol. Phys. 89, 736-744.

O'Carroll, D., Erhardt, S., Pagani, M., and Barton, S.C. (2001). The Polycomb -Group Gene Ezh2 Is Required for Early Mouse Development. Mol. Cell. Biol. 21, 4330-4336.

965 Oksuz, O., Narendra, V., Lee, C.H., Descostes, N., LeRoy, G., Raviram, R., Blumenberg, L., Karch, K., Rocha, P.P., Garcia, B.A., et al. (2018). Capturing the Onset of PRC2-Mediated Repressive Domain Formation. Mol. Cell 70, 1149-1162.e5.

Orlando, D.A., Chen, M.W., Brown, V.E., Solanki, S., Choi, Y.J., Olson, E.R., Fritz, C.C., 970 Bradner, J.E., and Guenther, M.G. (2014). Quantitative ChIP-Seq normalization reveals global modulation of the epigenome. Cell Rep. 9, 1163-1170.

Panagopoulos, I., Mertens, F., and Griffin, C.A. (2008). An endometrial stromal sarcoma cell line with the JAZF1/PHF1 chimera. Cancer Genet. Cytogenet. 185, 74-77.

Panagopoulos, I., Micci, F., Thorsen, J., Gorunova, L., Eibak, A.M., Bjerkehagen, B., Davidson, B., and Heim, S. (2012). Novel fusion of MYST/Esa1-associated factor 6 and PHF1 in endometrial stromal sarcoma. PLoS One 7, 3-8.

980 Pasini, D., Bracken, A.P., Jensen, M.R., Denchi, E.L., Helin, K., Birve, A., Sengupta, A., Beuchle, D., Larsson, J., Kennison, J., et al. (2004). Suz12 is essential for mouse development and for EZH2 histone methyltransferase activity. EMBO J. 23, 4061-4071.

Pasini, D., Bracken, A.P., Hansen, J.B., Capillo, M., and Helin, K. (2007). The Polycomb Group 985 Protein Suz12 Is Required for Embryonic Stem Cell Differentiation. Mol. Cell. Biol. 27, 37693779 .

Perino, M., van Mierlo, G., Karemaker, I.D., van Genesen, S., Vermeulen, M., Marks, H., van Heeringen, S.J., and Veenstra, G.J.C. (2018). MTF2 recruits Polycomb Repressive Complex 2 by 990 helical-shape-selective DNA binding. Nat. Genet. 50, 1002-1010.

Piunti, A., Smith, E.R., Morgan, M.A.J., Ugarenko, M., Khaltyan, N., Helmin, K.A., Ryan, C.A., Murray, D.C., Rickels, R.A., Yilmaz, B.D., et al. (2019). Catacomb: An endogenous inducible gene that antagonizes H3K27 methylation activity of Polycomb repressive complex 2 via an 
H3K27M-like mechanism. Sci. Adv. 5. 2375-2548.

Prezioso, C., and Orlando, V. (2011). Polycomb proteins in mammalian cell differentiation and plasticity. FEBS Lett. 585, 2067-2077.

1000 Procida, T., Friedrich, T., Jack, A.P.M., Peritore, M., Bönisch, C., Christian Eberl, H., Daus, N., Kletenkov, K., Nist, A., Stiewe, T., et al. (2021). Jazf1, a novel p400/tip60/nua4 complex member, regulates h2a.Z acetylation at regulatory regions. Int. J. Mol. Sci. 22, 1-20.

Przybyl, J., Kidzinski, L., Hastie, T., Debiec-Rychter, M., Nusse, R., and van de Rijn, M. (2018). 1005 Gene expression profiling of low-grade endometrial stromal sarcoma indicates fusion proteinmediated activation of the Wnt signaling pathway. Gynecol. Oncol. 149, 388-393.

Quinlan, A.R., and Hall, I.M. (2010). BEDTools: A flexible suite of utilities for comparing genomic features. Bioinformatics 26, 841-842.

Ragazzini, R., Pérez-Palacios, R., Baymaz, I.H., Diop, S., Ancelin, K., Zielinski, D., Michaud, A., Givelet, M., Borsos, M., Aflaki, S., et al. (2019). EZHIP constrains Polycomb Repressive Complex 2 activity in germ cells. Nat. Commun. 10, 3858.

1015 Ramírez, F., Dündar, F., Diehl, S., Grüning, B.A., and Manke, T. (2014). DeepTools: A flexible platform for exploring deep-sequencing data. Nucleic Acids Res. 42, 187-191.

Riising, E.M., Comet, I., Leblanc, B., Wu, X., Johansen, J.V., and Helin, K. (2014). Gene silencing triggers polycomb repressive complex 2 recruitment to $\mathrm{CpG}$ Islands genome wide. Mol. Cell 55, $1020 \quad 347-360$.

Raudvere, U., Kolberg, L., Kuzmin, I., Arak, T., Adler, P., Peterson, H. and Vilo, J. (2019). g:Profiler: a web server for functional enrichment analysis and conversions of gene list. Nucleic Acids Res. doi:10.1093/nar/gkz369

Ross-Innes, C.S., Stark, R., Teschendorff, A.E., Holmes, K.A., Ali, H.R., Dunning, M.J., Brown, G.D., Gojis, O., Ellis, I.O., Green, A.R., et al. (2012). Differential oestrogen receptor binding is associated with clinical outcome in breast cancer. Nature 481, 389-393.

1030 Sanulli, S., Justin, N., Teissandier, A., Ancelin, K., Portoso, M., Caron, M., Michaud, A., Lombard, B., da Rocha, S.T., Offer, J., et al. (2015). Jarid2 Methylation via the PRC2 Complex Regulates H3K27me3 Deposition during Cell Differentiation. Mol. Cell 57, 769-783.

Scaffidi, P., and Misteli, T. (2011). In vitro generation of human cells with cancer stem cell 1035 properties. Nat Cell Biol 13, 1051-61.

Schuettengruber, B., Bourbon, H.M., Di Croce, L., and Cavalli, G. (2017). Genome Regulation by Polycomb and Trithorax: 70 Years and Counting. Cell 171,34-57.

1040 Shen, X., Kim, W., Fujiwara, Y., Simon, M.D., Liu, Y., Mysliwiec, M.R., Yuan, G., Lee, Y., and 
Orkin, S.H. (2009). Jumonji Modulates Polycomb Activity and Self-Renewal versus Differentiation of Stem Cells. Cell 139, 1303-1314.

Steunou, A.L., Rossetto, D., and Côté, J. (2014). Regulating Chromatin by Histone Acetylation. In Fundamentals of Chromatin, (New York, NY: Springer New York), pp. 147-212.

Walker, E., Chang, W.Y., Hunkapiller, J., Cagney, G., Garcha, K., Torchia, J., Krogan, N.J., Reiter, J.F., and Stanford, W.L. (2010). Polycomb-like 2 Associates with PRC2 and Regulates Transcriptional Networks during Mouse Embryonic Stem Cell Self-Renewal and Differentiation. 1050 Cell Stem Cell 6, 153-166.

Wang, X., Paucek, R.D., Gooding, A.R., Brown, Z.Z., Ge, E.J., Muir, T.W., and Cech, T.R. (2017a). Molecular analysis of PRC2 recruitment to DNA in chromatin and its inhibition by RNA. Nat. Struct. Mol. Biol. 24, 1028-1038.

Wang, X., Goodrich, K.J., Gooding, A.R., Naeem, H., Archer, S., Paucek, R.D., Youmans, D.T., Cech, T.R., and Davidovich, C. (2017b). Targeting of Polycomb Repressive Complex 2 to RNA by Short Repeats of Consecutive Guanines. Mol. Cell 65, 1056-1067.e5.

1060 Xu, Y., Ayrapetov, M.K., Xu, C., Gursoy-Yuzugullu, O., Hu, Y., and Price, B.D. (2012). Histone H2A.Z Controls a Critical Chromatin Remodeling Step Required for DNA Double-Strand Break Repair. Mol. Cell 48, 723-733.

Youmans, D.T., Schmidt, J.C., and Cech, T.R. (2018). Live-cell imaging reveals the dynamics of 1065 PRC2 and recruitment to chromatin by SUZ12-associated subunits. Genes Dev. 32, 794-805.

Zhang, Q., McKenzie, N.J., Warneford-Thomson, R., Gail, E.H., Flanigan, S.F., Owen, B.M., Lauman, R., Levina, V., Garcia, B.A., Schittenhelm, R.B., et al. (2019). RNA exploits an exposed regulatory site to inhibit the enzymatic activity of PRC2. Nat. Struct. Mol. Biol. 26, 237-247.

Zhang, Q., Agius, S.C., Flanigan, S.F., Levina, V., Owen, B.M., and Davidovich, C. (2020). Convergent evolution between PALI1 and JARID2 for the allosteric activation of PRC2. BioRxiv $1,1-35$.

1075 Zhang, Z., Jones, A., Sun, C.W., Li, C., Chang, C.W., Joo, H.Y., Dai, Q., Mysliwiec, M.R., Wu, L.C., Gou, Y., et al. (2011). PRC2 complexes with JARID2, MTF2, and esPRC2p48 in ES cells to modulate ES cell pluripotency and somatic cell reprograming. Stem Cells 29, 229-240.

Zhao, J., Ohsumi, T.K., Kung, J.T., Ogawa, Y., Grau, D.J., Sarma, K., Song, J.J., Kingston, R.E., 1080 Borowsky, M., and Lee, J.T. (2010). Genome-wide Identification of Polycomb-Associated RNAs by RIP-seq. Mol. Cell 40, 939-953.

Zoabi, M., Nadar-Ponniah, P.T., Khoury-Haddad, H., Usaj, M., Budowski-Tal, I., Haran, T., Henn, A., Mandel-Gutfreund, Y., and Ayoub, N. (2014). RNA-dependent chromatin localization of 1085 KDM4D lysine demethylase promotes H3K9me3 demethylation. Nucleic Acids Res. 42, 1302613038. 
a

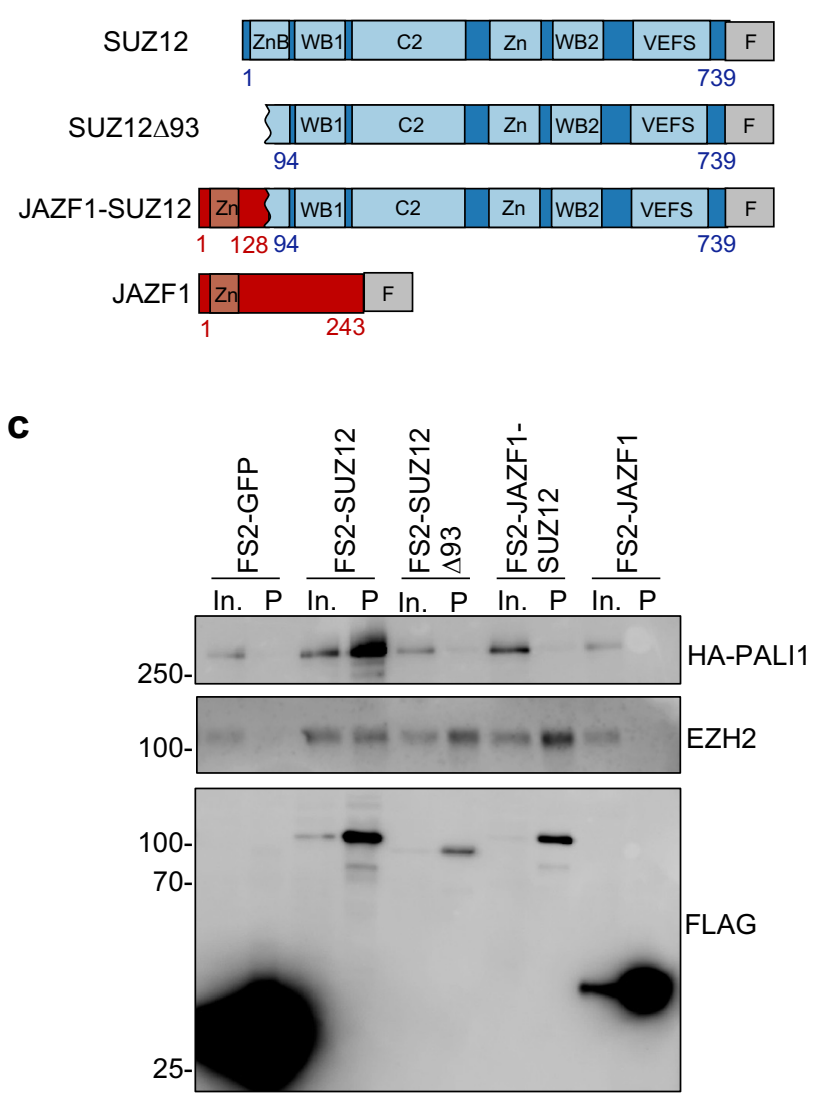

b

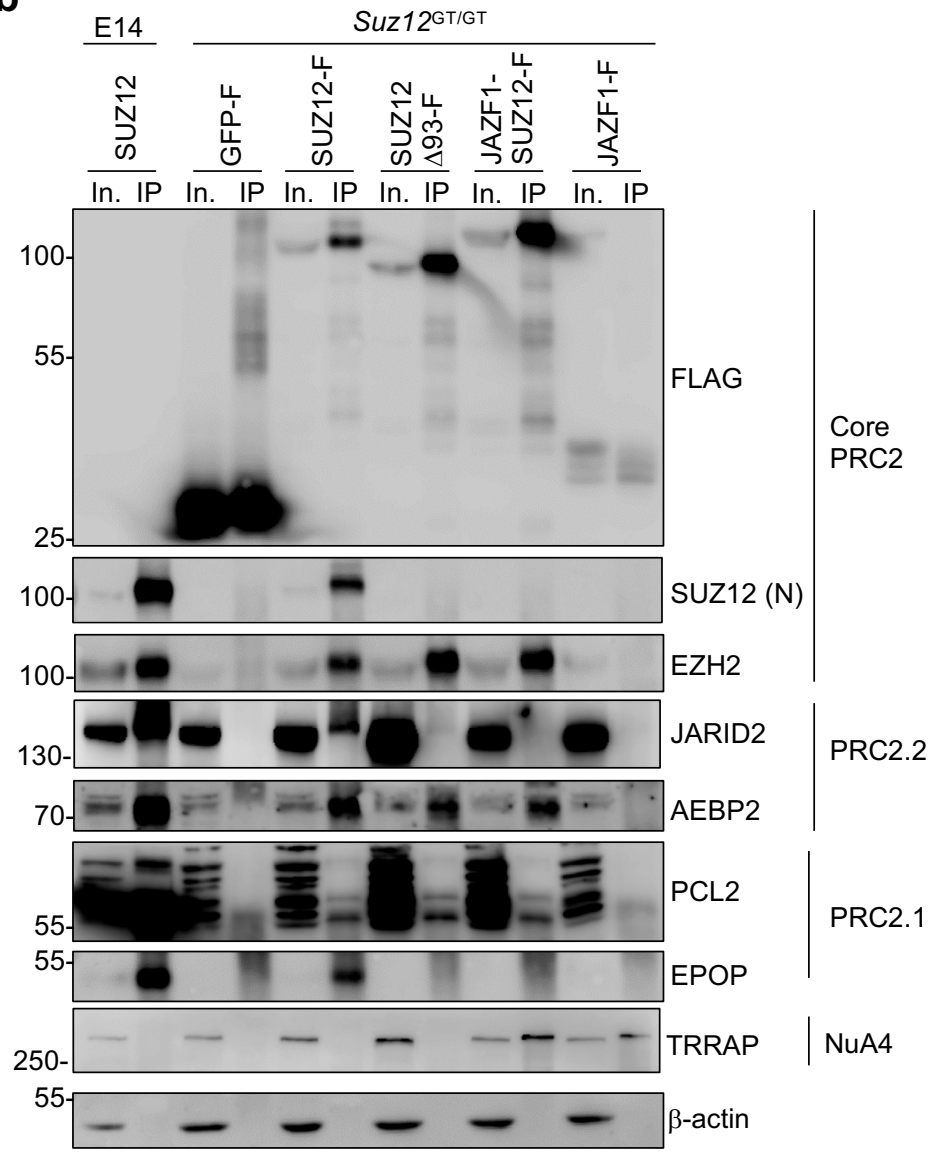

Figure 1. JAZF1-SUZ12 lacks interaction with JARID2, EPOP and PALI1 due to loss of the SUZ12 N-terminus.

a. Models of SUZ12, SUZ12 $\triangle 93$, JAZF1-SUZ12 and JAZF1 constructs with C-terminal FLAG tags $(F)$.

b. Immunoblots for FLAG, SUZ12 (N-terminus), EZH2, JARID2, AEBP2, PCL2, EPOP, TRRAP and $\beta$-actin in input (In.) and SUZ12 IPs from WT E14 cells or input and FLAG IPs from Suz12GT/GT cells stably expressing FLAG-tagged GFP, SUZ12, SUZ12 $\triangle 93$, JAZF1-SUZ12 or JAZF1.

c. Immunoblots for FLAG, HA-PALI and EZH2 in input and Strep-Tactin pull-downs (P) from NIH3T3 cells transfected with HA-tagged PALI1 and FS2-tagged GFP, SUZ12, SUZ12 $\triangle 93$, JAZF1SUZ12 or JAZF1.

See also Figure $\mathrm{S} 1$. 

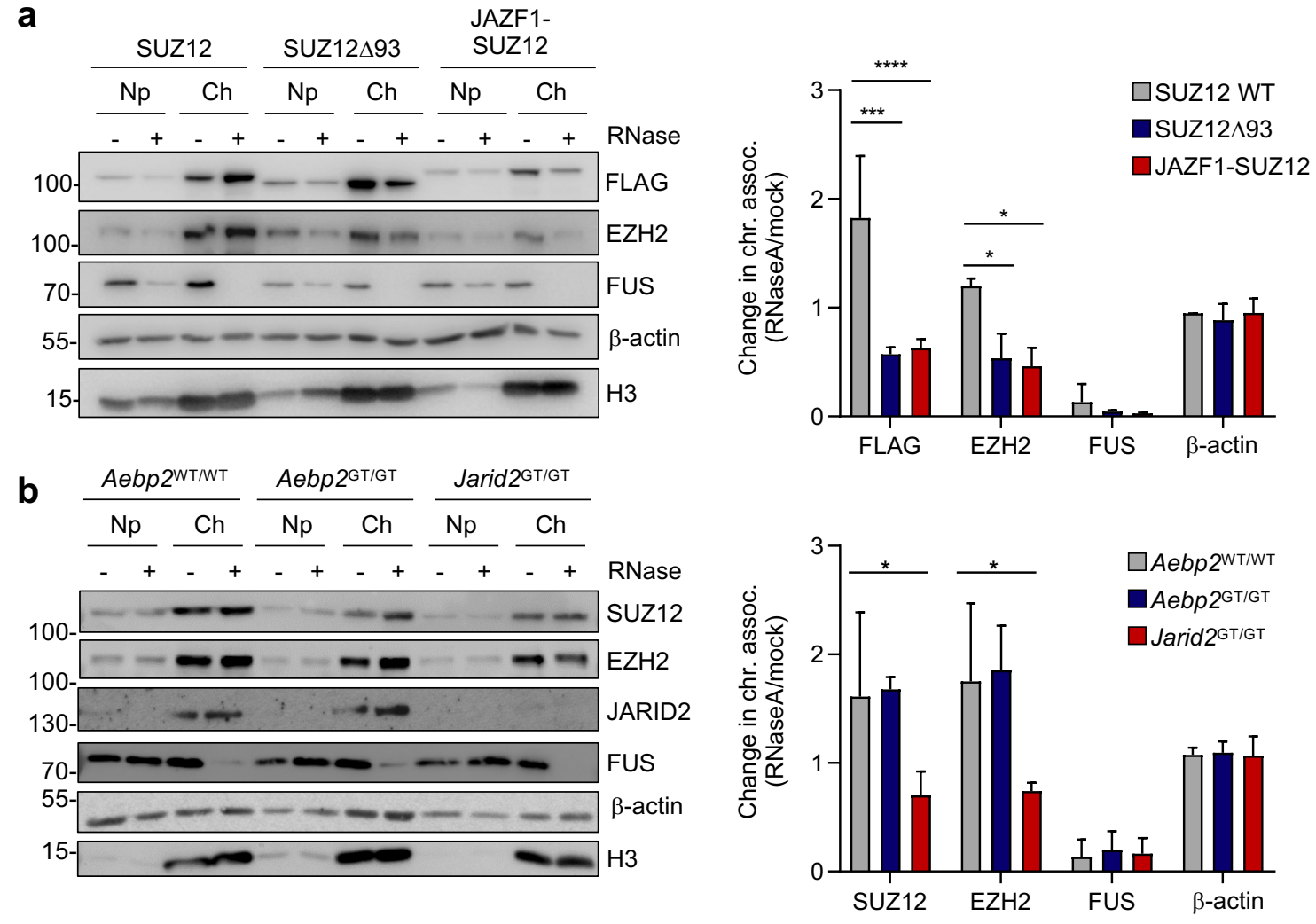

C
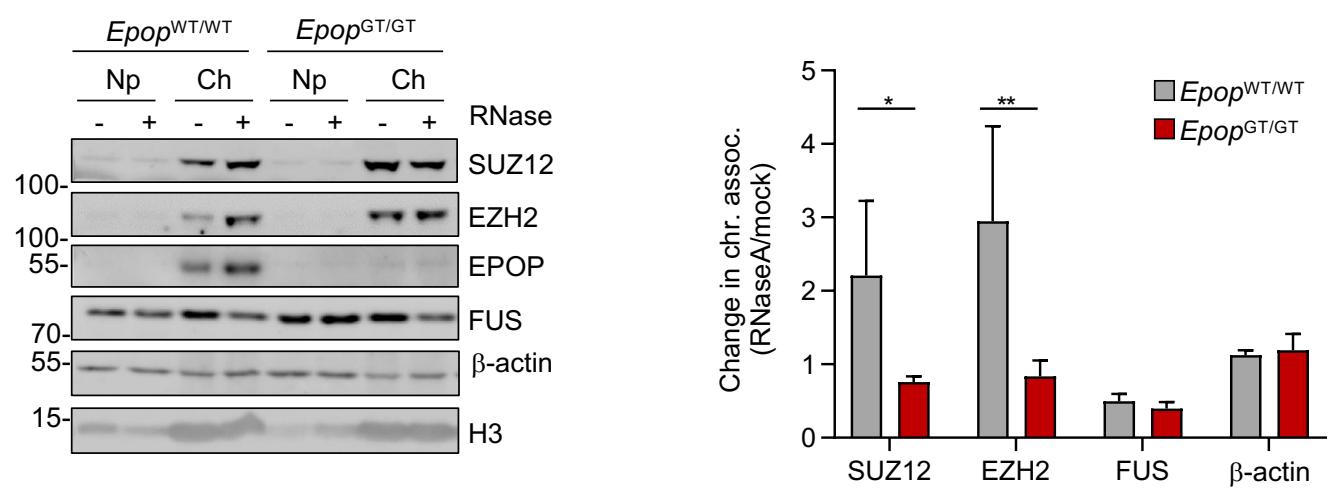

Figure 2. JAZF1-SUZ12 is not recruited to chromatin upon RNA depletion due to loss of interaction with JARID2 and EPOP.

a. Left: Immunoblots for FLAG, EZH2, FUS, $\beta$-actin and $\mathrm{H} 3$ in nucleoplasm (Np) and chromatin (Ch) fractions from mock or RNaseA-treated Suz12 ${ }^{\mathrm{GT} / \mathrm{GT}}$ cells expressing either FLAG-tagged SUZ12, SUZ12 $\triangle 93$ or JAZF1-SUZ12. Representative of three independent experiments. Right: Fold change in FLAG, EZH2, FUS and $\beta$-actin in the chromatin fraction upon RNaseA treatment (mean and s.d, $\mathrm{n}=3$ ).

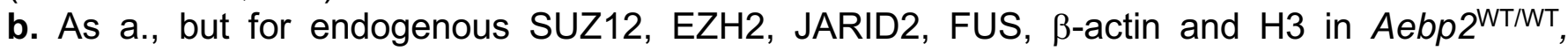
Aebp2 ${ }^{\mathrm{GT} / \mathrm{GT}}$ and Jarid2 ${ }^{\mathrm{GT} / \mathrm{GT}} \mathrm{ESC}$.

c. As a., but for endogenous SUZ12, EZH2, EPOP, FUS, $\beta$-actin and H3 in Epop ${ }^{\mathrm{WT} / \mathrm{WT}}$ and Epop ${ }^{\mathrm{GT} / \mathrm{GT}}$ ESC.

See also Figure S2. 

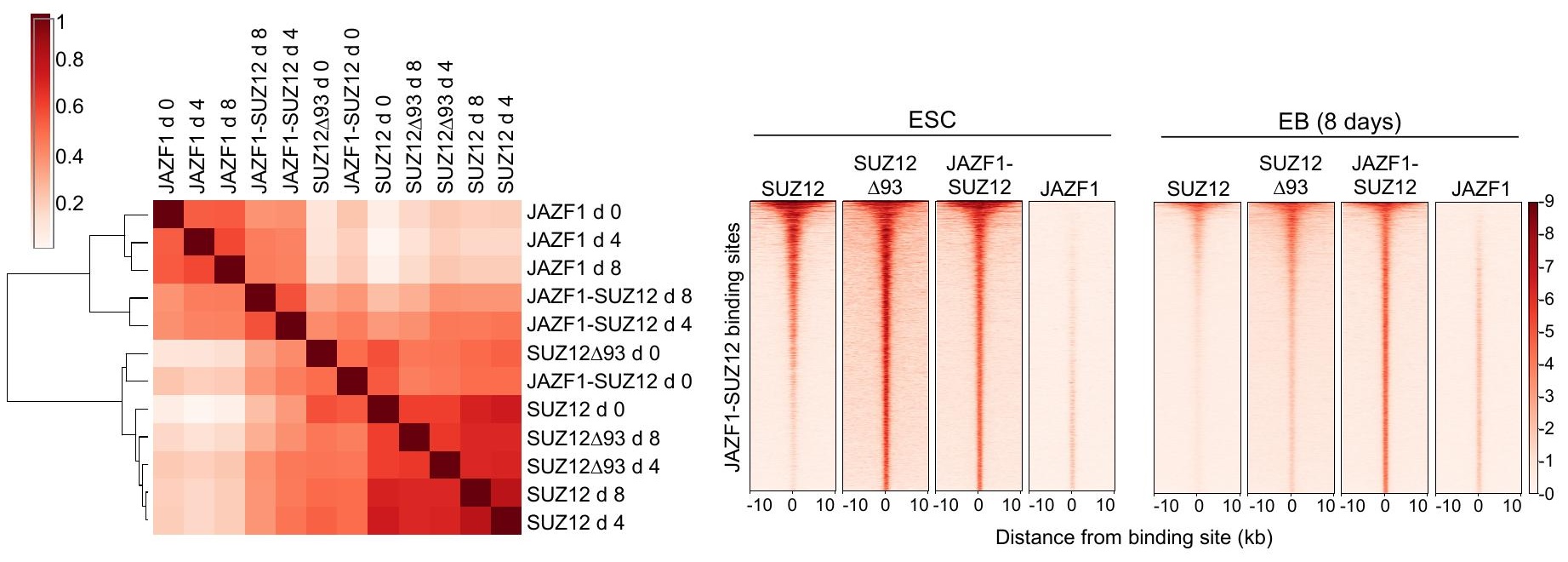

C

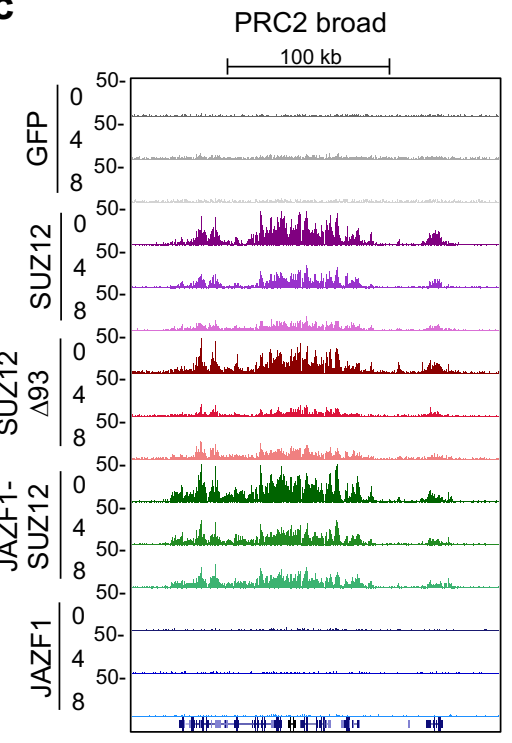

Hoxa cluster

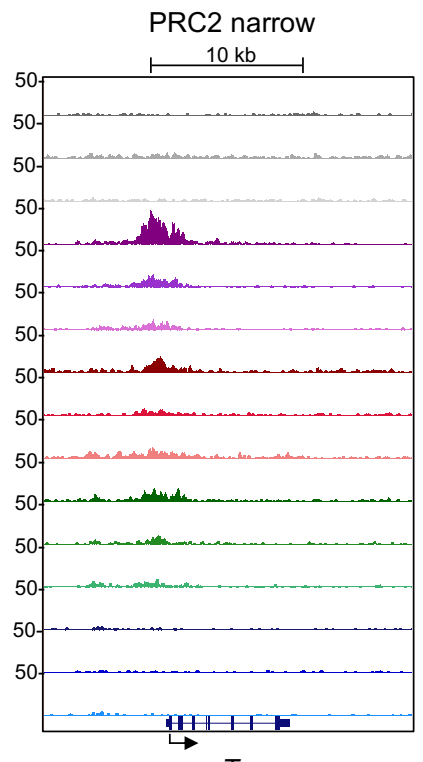

$T$

$\circ \infty$
要 $\frac{\pi}{0}$ $0 \infty \stackrel{N}{\sim} \circ \infty$

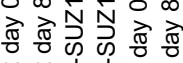

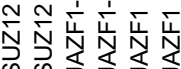

GO:0007275 multicellular organism development GO:0007267 cell-cell signalling GO:0003700 DNA-binding transcription factor activity GO:0016477 cell migration GO:0016055 Wnt signaling pathway GO:0007155 cell adhesion GO:0006935 chemotaxis GO:0007165 signal transduction GO:0048566 embryonic digestive tract development GO:0008092 cytoskeletal protein binding GO:0006914 autophagy GO:0008361 regulation of cell size GO:0044267 cellular protein metabolic process GO:0051641 cellular localization GO:0051726 regulation of cell cycle GO:0006396 RNA processing

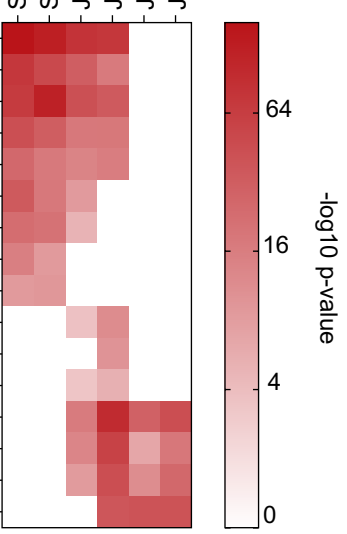

JAZF1-SUZ12 and JAZF1

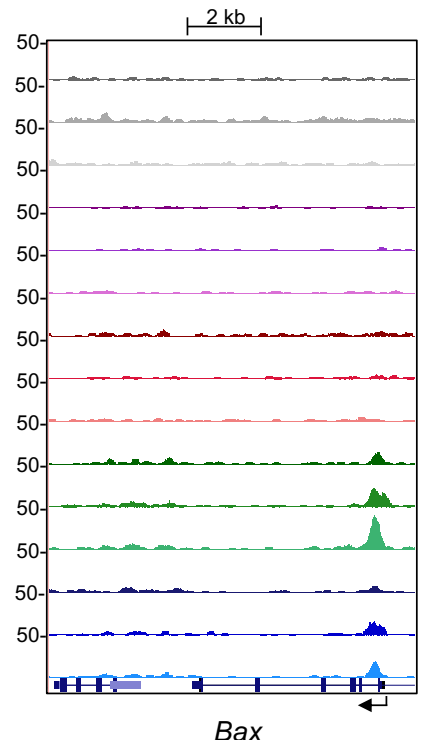

Bax
JAZF1-SUZ12 specific

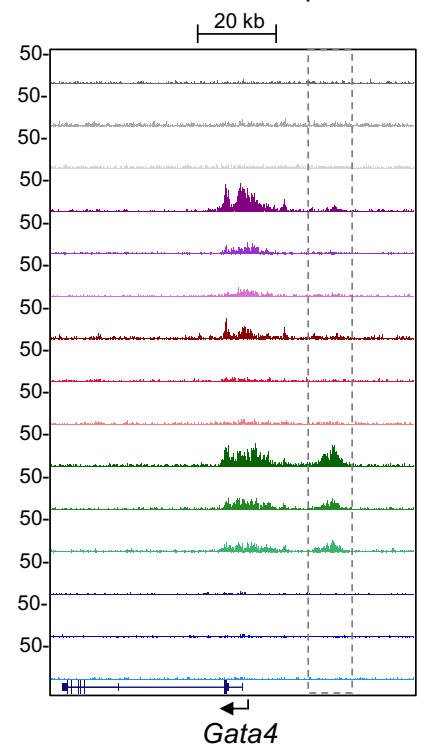


Figure 3. JAZF1-SUZ12 takes on a JAZF1-like binding profile during cell differentiation

a. Hierarchical clustering showing the relationship between the patterns of SUZ12, SUZ12 $\triangle 93$, JAZF1-SUZ12 and JAZF1 genome occupancy in ESC and in embryoid bodies (EB) 4 and 8 days after induction of EB formation. Pearson correlations between samples are shown by colour, according to the scale on the left.

b. Heatmaps showing SUZ12, SUZ12 $\triangle 93$, JAZF1-SUZ12 and JAZF1 occupancy at sites bound by JAZF1-SUZ12 in ESC (day 0) and sites bound by JAZF1-SUZ12 in EBs (day 8). Occupancy (normalised reads) is indicated by color, according to the scale on the right. At each timepoint, sites are ordered by the average occupancy of all factors, from high to low.

c. Four patterns of SUZ12, SUZ12 $\triangle 93$, JAZF1-SUZ12 and JAZF1 genome occupancy (normalised reads per 10 bp window) in ESC at 0,4 and 8 days after initiation of differentiation into EBs. Left: a broad region of shared SUZ12, SUZ12 93 and JAZF1-SUZ12 occupancy at the Hoxa cluster. Center left: a narrow region of SUZ12 occupancy at $T$ that exhibits reduced SUZ12 $\triangle 93$ and JAZF1-SUZ12 binding. Center right: shared JAZF1-SUZ12 and JAZF1 occupancy at Bax. Right: JAZF1-SUZ12-specific binding upstream of Gata4 not shared by SUZ12 or JAZF1 (dashed box).

d. Heatmap showing enrichment $\left(-\log _{10} p\right.$-value) of representative $G O$ terms within the sets of genes occupied by SUZ12, JAZF1-SUZ12 or JAZF1 in ESC or EBs (day 8).

See also Figure S3. 


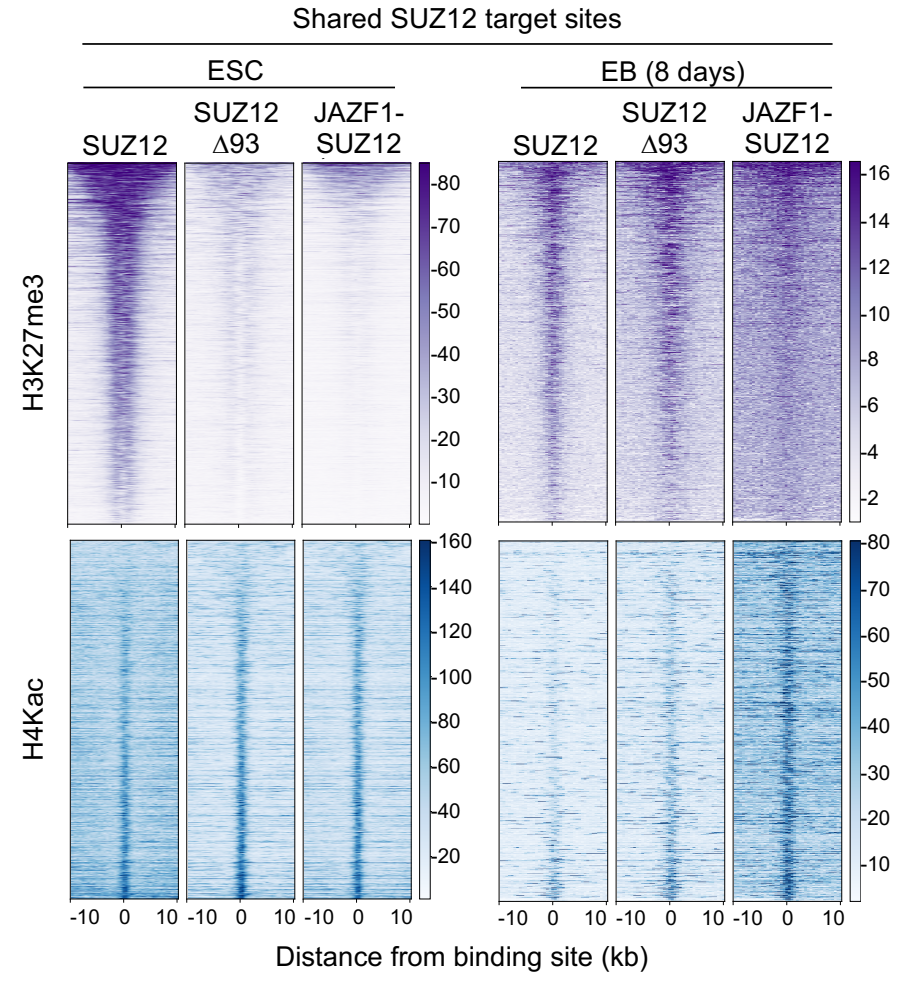

b

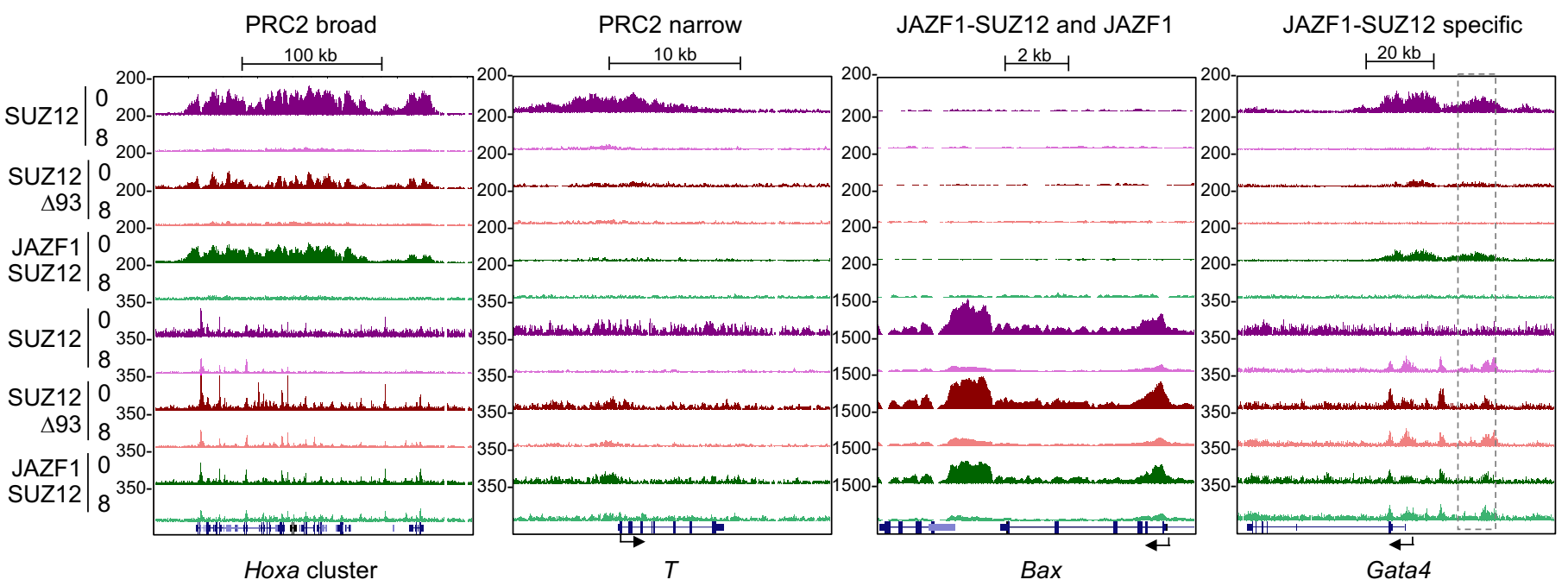

Figure 4. JAZF1-SUZ12 decreases H3K72me3 and increases H4Kac at PRC2 target genes.

a. Heatmaps of H3K27me3 (purple, top) and H4Kac (blue, bottom) around sites shared by SUZ12, SUZ12 $\triangle 93$ and JAZF1-SUZ12 in ESC and sites shared by the proteins in EB (day 8). Occupancy (normalised reads) is indicated by color, according to the scales on the right. At each timepoint, sites are ordered by H3K27me3 in cells expressing SUZ12, from high to low.

b. H3K27me3 and H4Kac (reads per 10bp window) at the regions shown in Figure 3C in ESC expressing SUZ12, SUZ12 $\triangle 93$ or JAZF1-SUZ12 at days 0 and 8 after initiation of differentiation into EBs. Dashed box upstream of GATA4 marks the region of ectopic JAZF1-SUZ12 binding from Figure 3C.

See also Figure S4. 

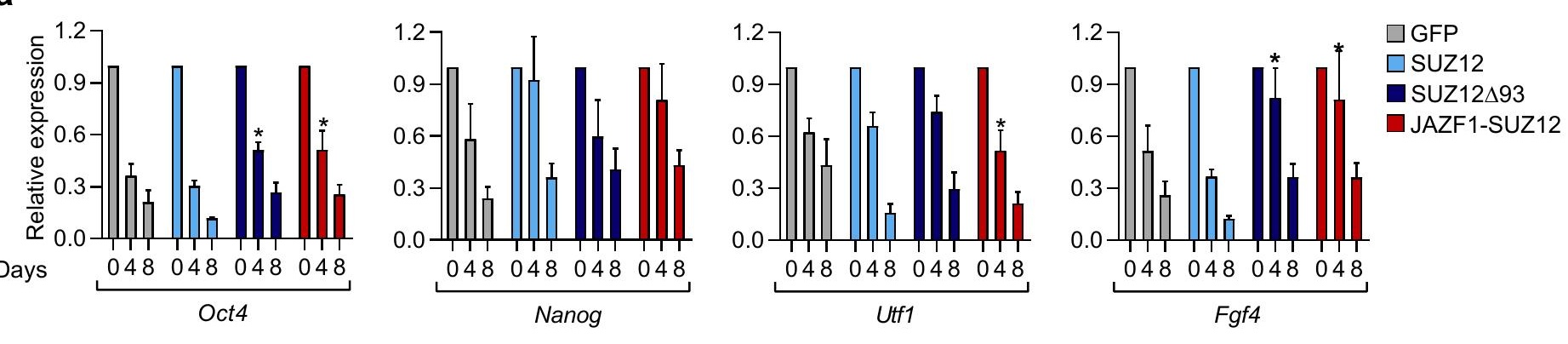

b
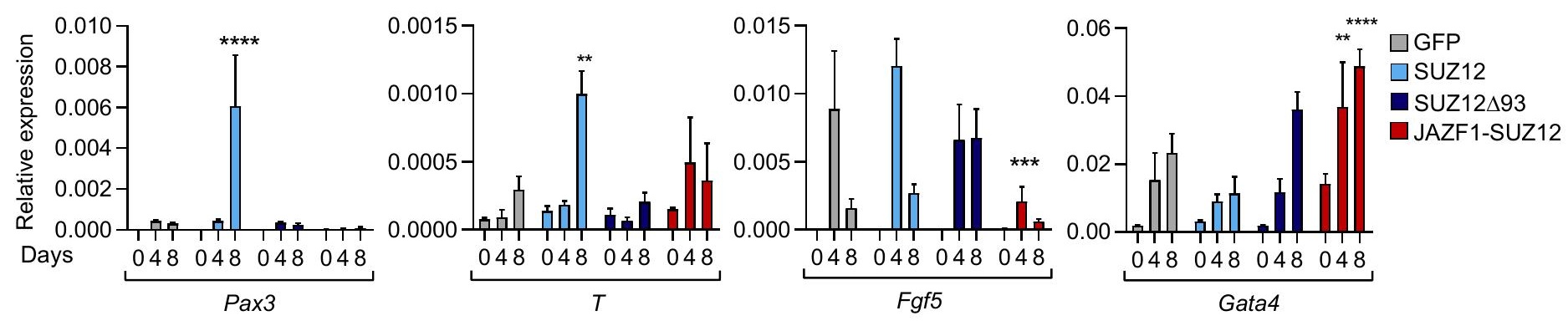

C

d
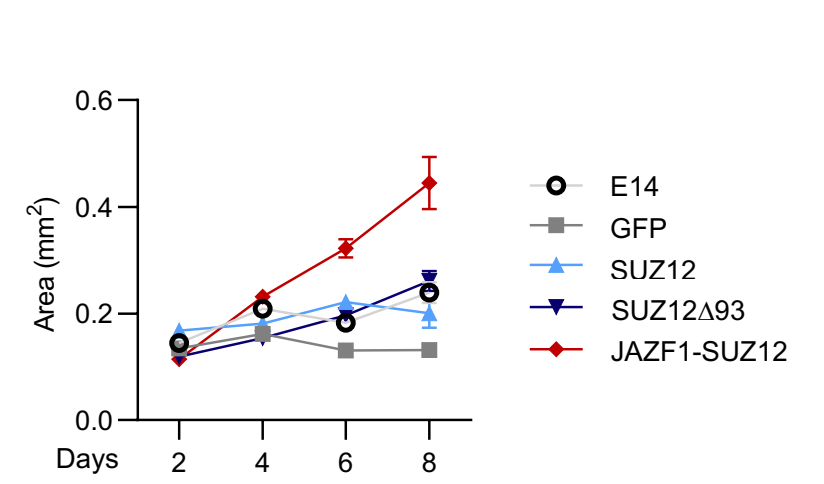

JAZF1-SUZ12
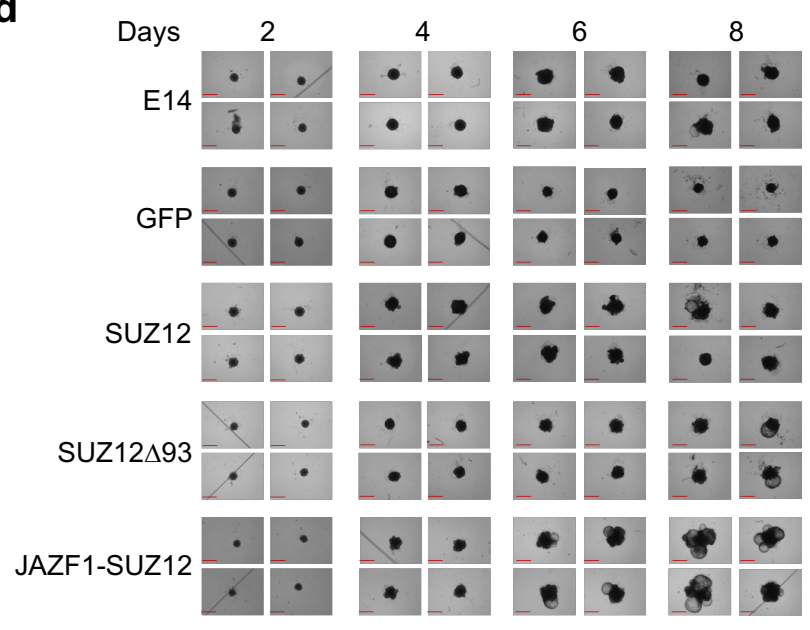

Figure 5. JAZF1-SUZ12 disrupts gene expression during cell differentiation.

a. Expression of Oct4, Nanog, Utf1 and Fgf4 (relative to Gapdh and day 0) at 0, 4 and 8 days after initiation of EB formation in Suz12 ${ }^{\mathrm{GT} / G T}$ ESC expressing GFP, SUZ12, SUZ12 $\triangle 93$ or JAZF1SUZ12 (mean and s.d. of 4 independent experiments; * $p<0.05$, 2-way ANOVA).

b. Expression of Pax3, T, Fgf5 and Gata4 (relative to Gapdh) at 0, 4 and 8 days after initiation of EB formation in Suz12 ${ }^{\text {GT/GT }}$ ESC expressing GFP, SUZ12, SUZ12 $\triangle 93$ or JAZF1-SUZ12 (mean and s.d. of 4 independent experiments; ${ }^{*} p<0.05$, 2-way ANOVA).

c. Size of EBs formed by WT E14 ESC and Suz12 ${ }^{\text {GT/GT }}$ ESC expressing GFP, SUZ12, SUZ12 $\triangle 93$ or JAZF1-SUZ12 measured at 2, 4, 6 and 8 days after initiation of EB formation (mean and s.d, $\mathrm{n} \geq 55$ ).

d. Representative images of Suz12 ${ }^{\mathrm{GT} / \mathrm{GT}}$ ESC expressing GFP, SUZ12, SUZ12 $\triangle 93$ or JAZF1SUZ12 at 2, 4, 6 and 8 days after initiation of EB formation. The red reference bars mark $0.65 \mathrm{~mm}$. 


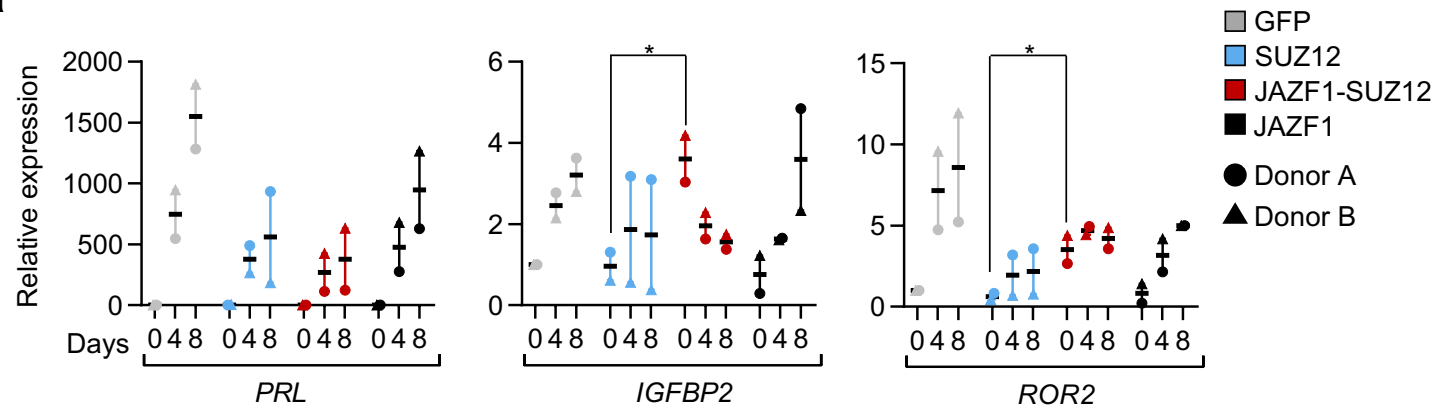

b

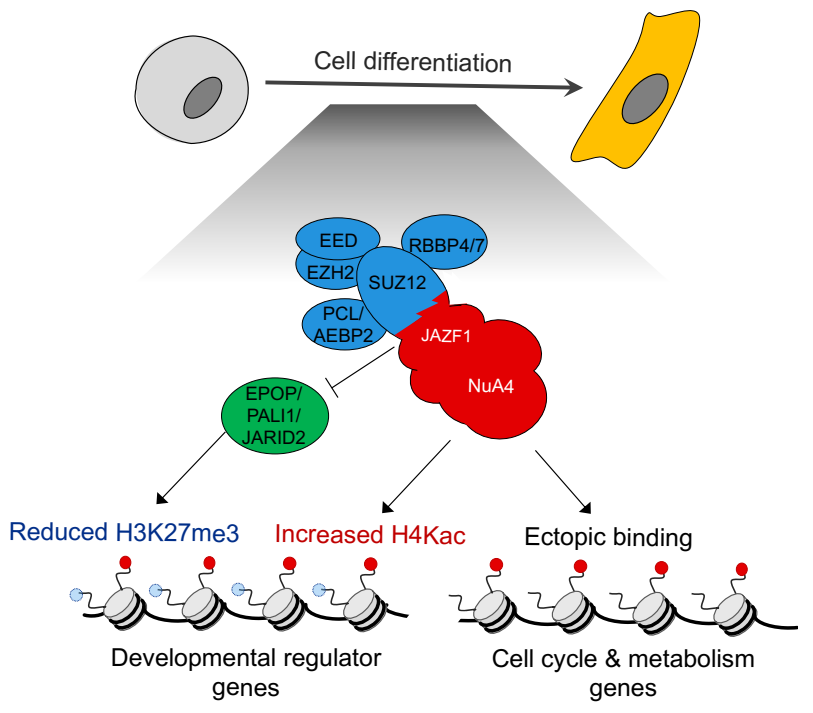

Figure 6. JAZF1-SUZ12 alters gene expression in primary human endometrial stromal cells (hEnSC).

a. Expression of PRL, IGFBP2 and ROR2 (relative to 5S rRNA and GFP day 0 ) at 0,4 and 8 days after initiating decidualisation of hEnSC expressing GFP, SUZ12, JAZF1-SUZ12 or JAZF1 (mean and s.d., 2 donors, ${ }^{*} \mathrm{p}<0.05$, t-test).

b. Model: Loss of the SUZ12 N-terminus prevents interaction of JAZF1-SUZ12 with EPOP, PALI1 and JARID2, while fusion to JAZF1 induces association with NuA4/TIP60. Loss of interaction with EPOP, PALI1 and JARID2 decreases PRC2 occupancy at its target genes and reduces $\mathrm{H} 3 \mathrm{~K} 27 \mathrm{me} 3$, while fusion to JAZF1 increases $\mathrm{H} 4 \mathrm{Kac}$ at these sites during cell differentiation. Fusion to JAZF1 also triggers ectopic SUZ12 recruitment to functional classes of gene with which it does not normally associate. These changes in chromatin occupancy and histone modification are accompanied by dysregulated gene expression and defects in cell differentiation.

See also Figure S5. 

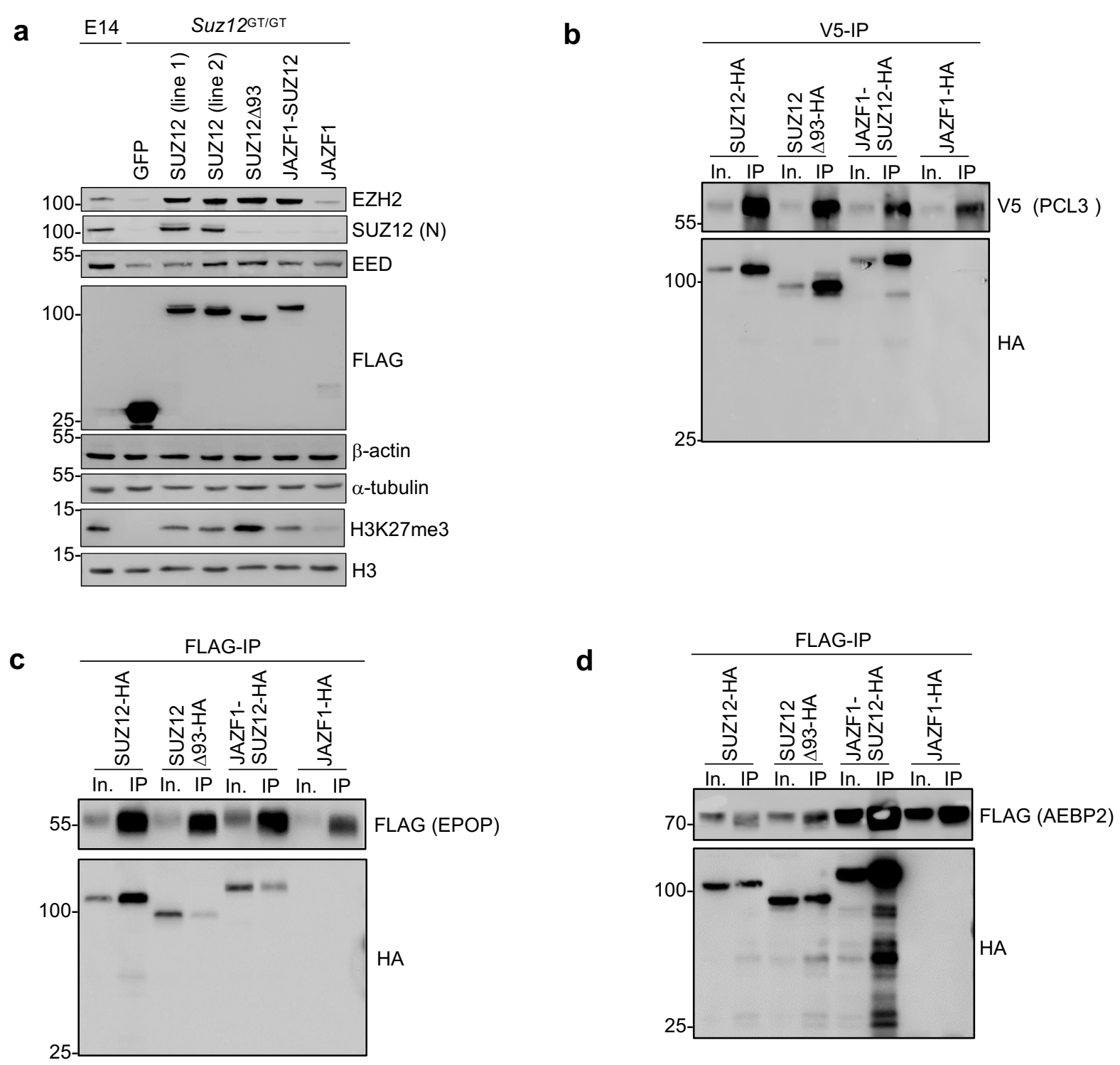

Figure S1. JAZF1-SUZ12 interacts with PCL3 and AEBP2 but shows reduced interaction with EPOP.

a. Levels of FLAG-tagged GFP, SUZ12 (2 different clones), SUZ12 993 , JAZF1-SUZ12 and JAZF1 in stable Suz12GT/GT cell lines in comparison to WT E14 cells.

b. Immunoblots for V5 and HA in input (In.) and SUZ12 IP fractions from NIH-3T3 cells cotransfected with V5-tagged PCL3 and HA-tagged SUZ12/JAZF1 constructs.

c. As b., except in cells transfected with FS2-EPOP and HA-tagged SUZ12/JAZF1 constructs.

d. As b., except in cells transfected with FS2-AEBP2 and HA-tagged SUZ12/JAZF1 constructs. 
a
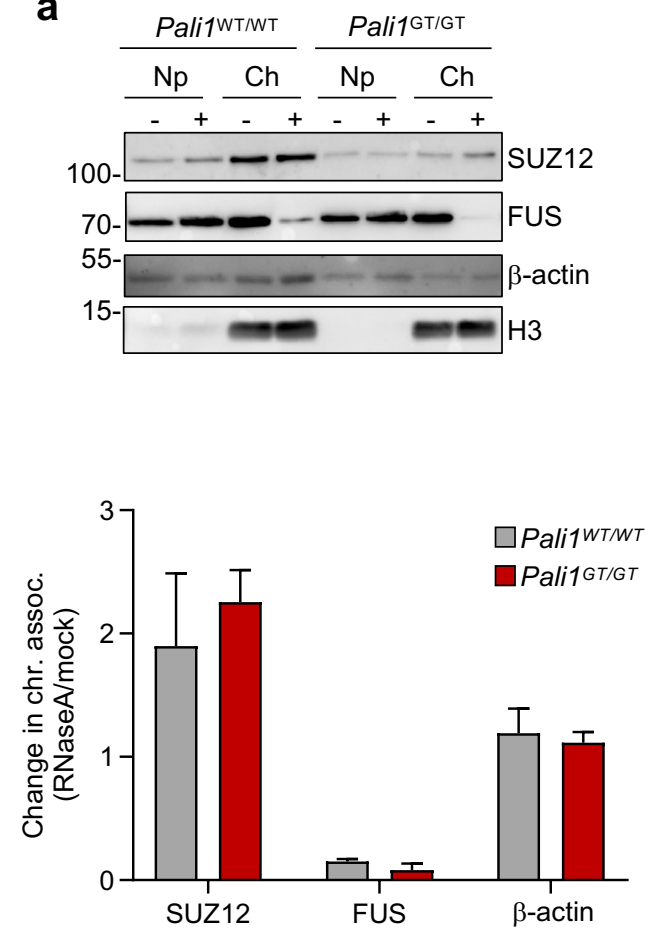

b
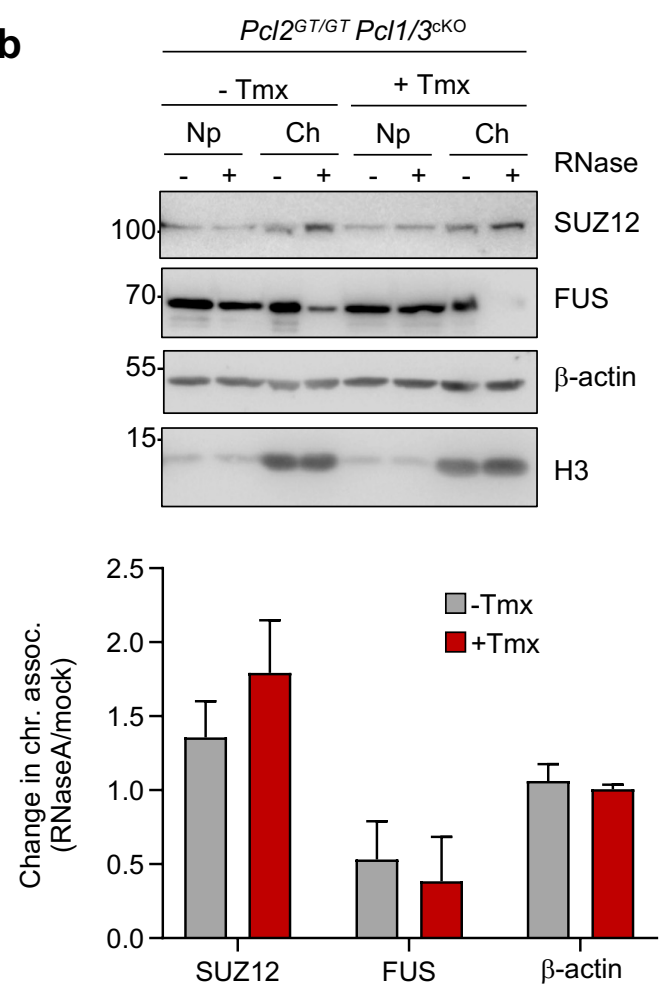

Figure S2. PALI1 and PCL1-3 are not required for recruitment of PRC2 to chromatin upon RNA depletion

a. Left: Immunoblots for SUZ12, FUS, $\beta$-actin, and H3 in nucleoplasm (Np) and chromatin (Ch) fractions from mock or RNaseA-treated WT or Pali1GT/GT cells. Representative of two independent experiments. Right: Fold change in FLAG, FUS and $\beta$-actin in the chromatin fraction upon RNaseA treatment (mean and s.d, $n=2$ )

b. Immunoblots for SUZ12, FUS, $\beta$-actin and $\mathrm{H} 3$ in nucleoplasm (Np) and chromatin (Ch) fractions from mock or RNaseA-treated Pcl2 ${ }^{\mathrm{GT} / \mathrm{GT}} P \mathrm{P} / 1 / 3^{\mathrm{cKO}}$ cells pre-treated or not with 4hydroxytamoxifen (tmx) to induce deletion of $P c / 1$ and $P c / 3$ (mean and s.d., $\mathrm{n}=2$ ). 
a

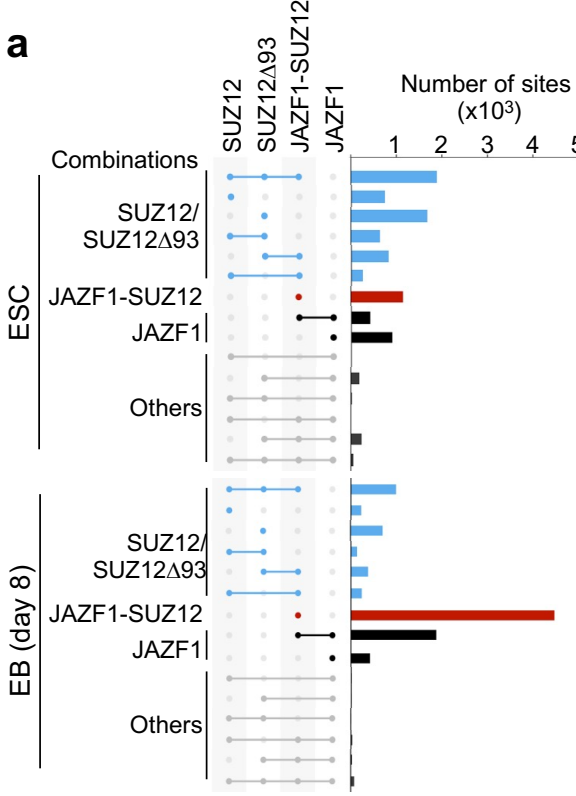

C
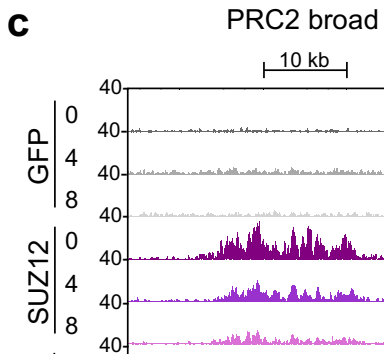

ธัง

文
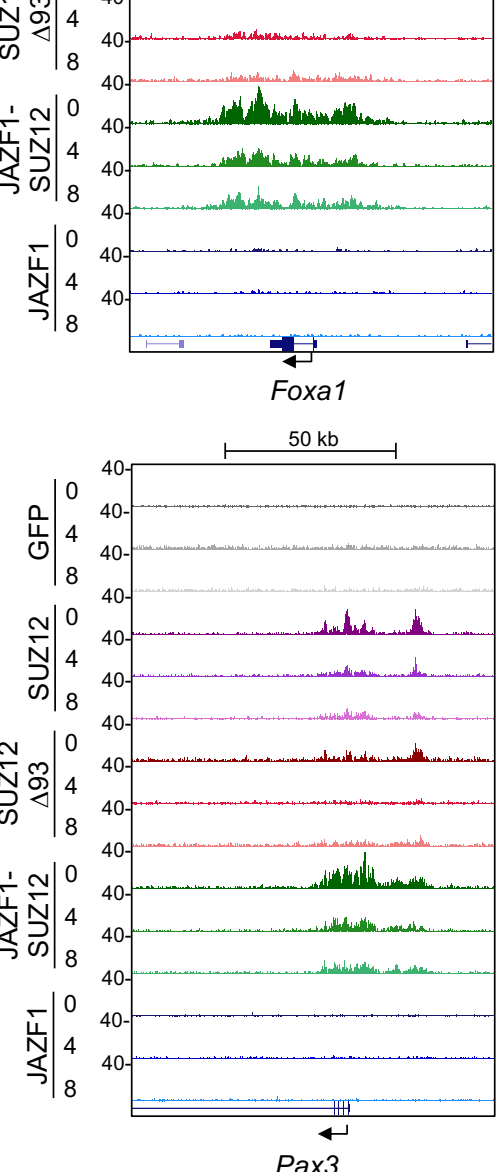

Pax3

b
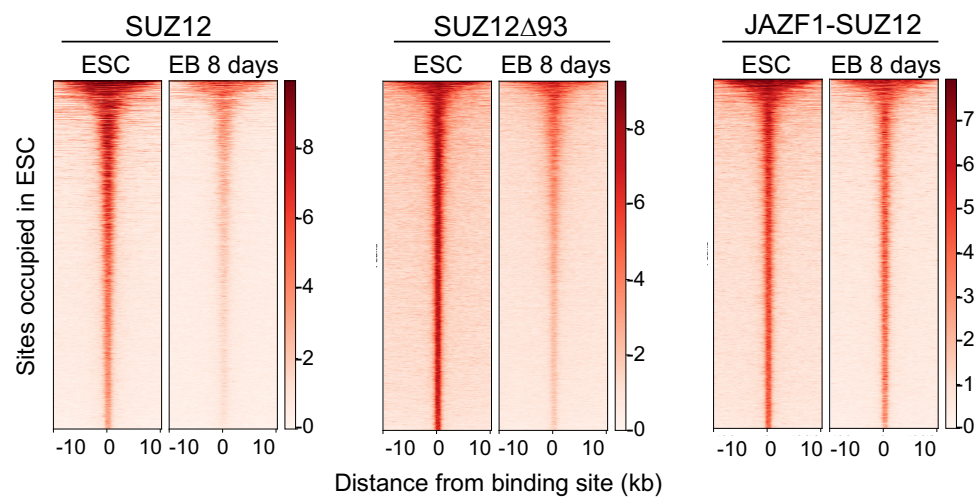

JAZF1-SUZ12 and JAZF1

JAZF1-SUZ12 specific (d4 and 8)

PRC2 narrow
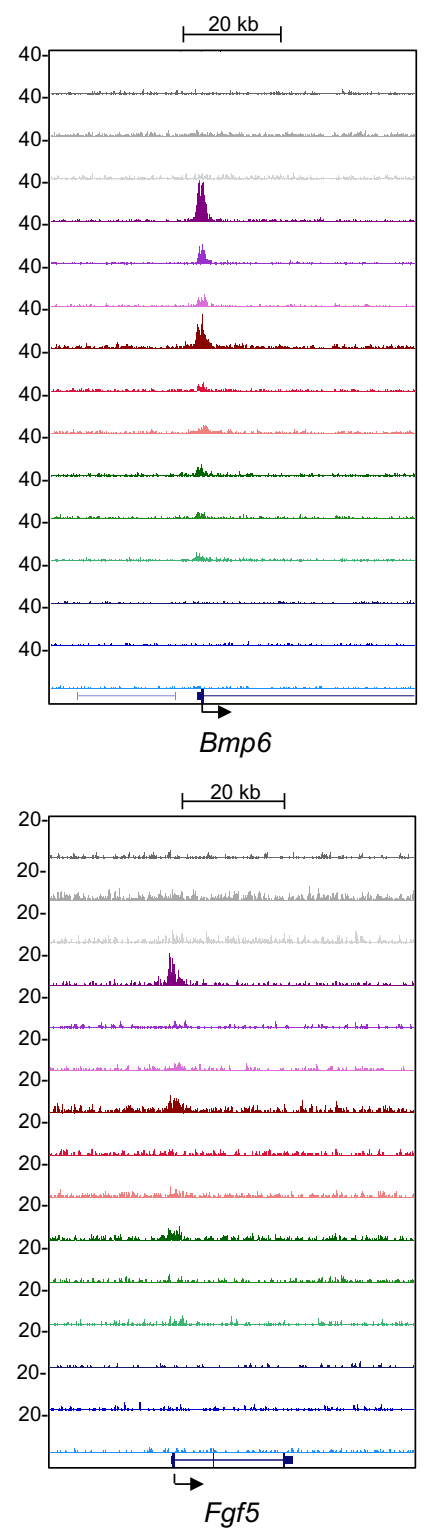

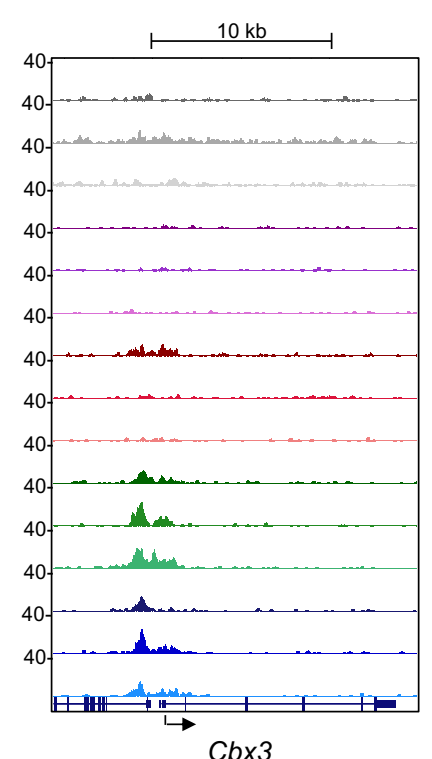

Cbx3

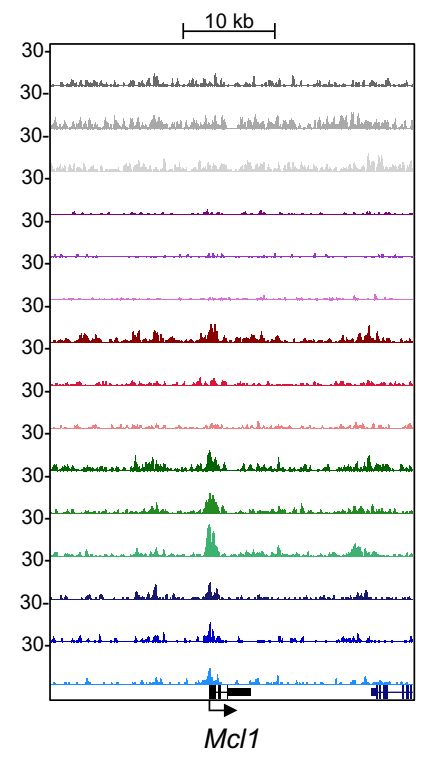

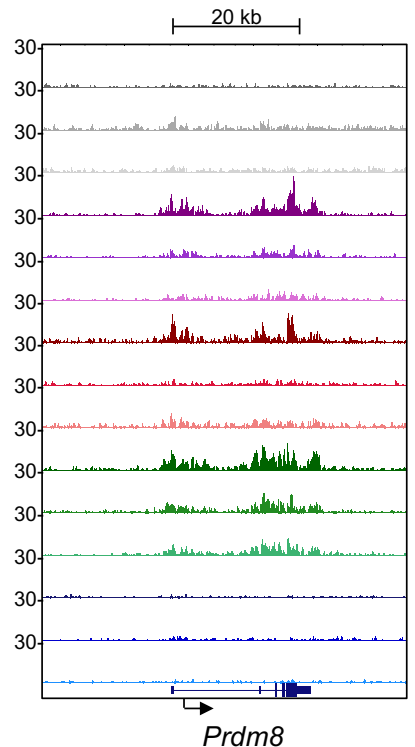

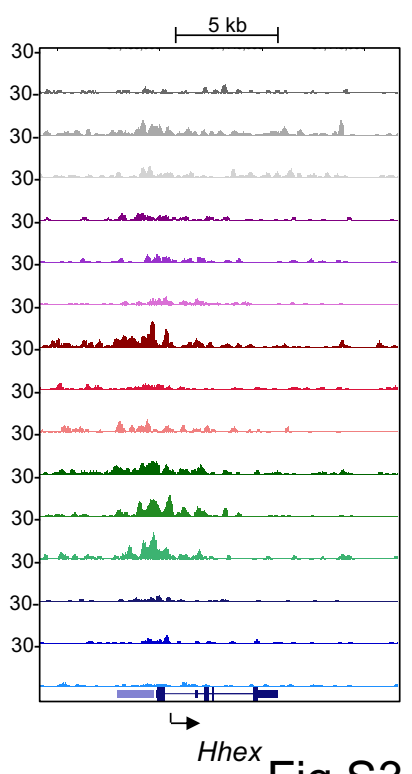


Figure S3. Differences in chromatin occupancy between JAZF1-SUZ12 and SUZ12.

a. UpSet plots showing the number of sites bound by different combinations of proteins in ESC and in EBs (8 days after induction of ESC differentiation). Binding combinations are grouped into sites bound by SUZ12 and/or SUZ12 $\triangle 93$ (light blue), sites bound by JAZF1-SUZ12 alone (red), sites bound by JAZF1-SUZ12 and/or JAF1 (black), and other combinations (grey).

b. Heatmaps showing SUZ12, SUZ12 93 or JAZF1-SUZ12 occupancy in ESC and day 8 EBs at sites occupied by each factor in ESC. Occupancy (normalised reads) is indicated by color, according to the scales on the right. For each factor, binding sites are ordered by occupancy in ESC, from high to low.

c. Further examples of the 4 patterns of JAZF1-SUZ12 occupancy shown in Figure 3C. Left: broad regions of SUZ12 binding at Foxa1 and Pax3. Centre left: narrow regions of SUZ12 binding at Bmp6 and Fgf5. Center right: Shared JAZF1-SUZ12 and JAZF1 occupancy at Cbx3 and Mcl1. Right: JAZF1-SUZ12-specific binding at Prdm8 and Hhex in day 4 and day 8 EBs. 

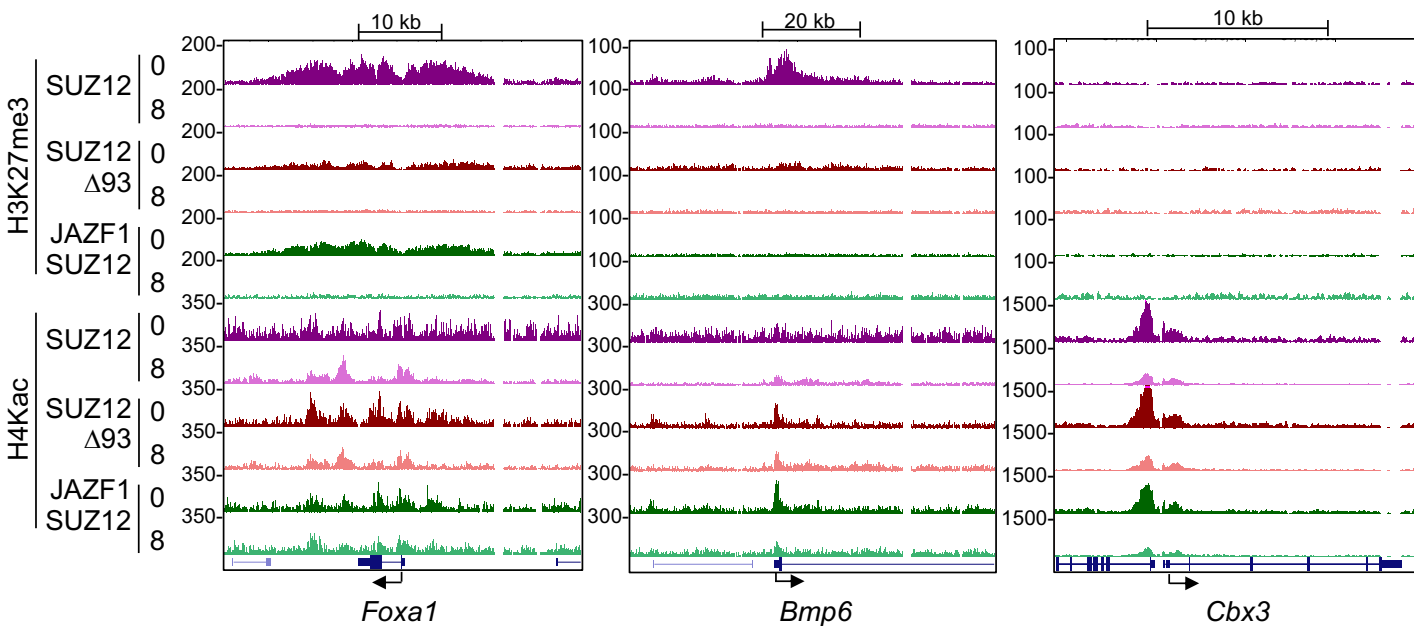

Foxa1

Cbx3
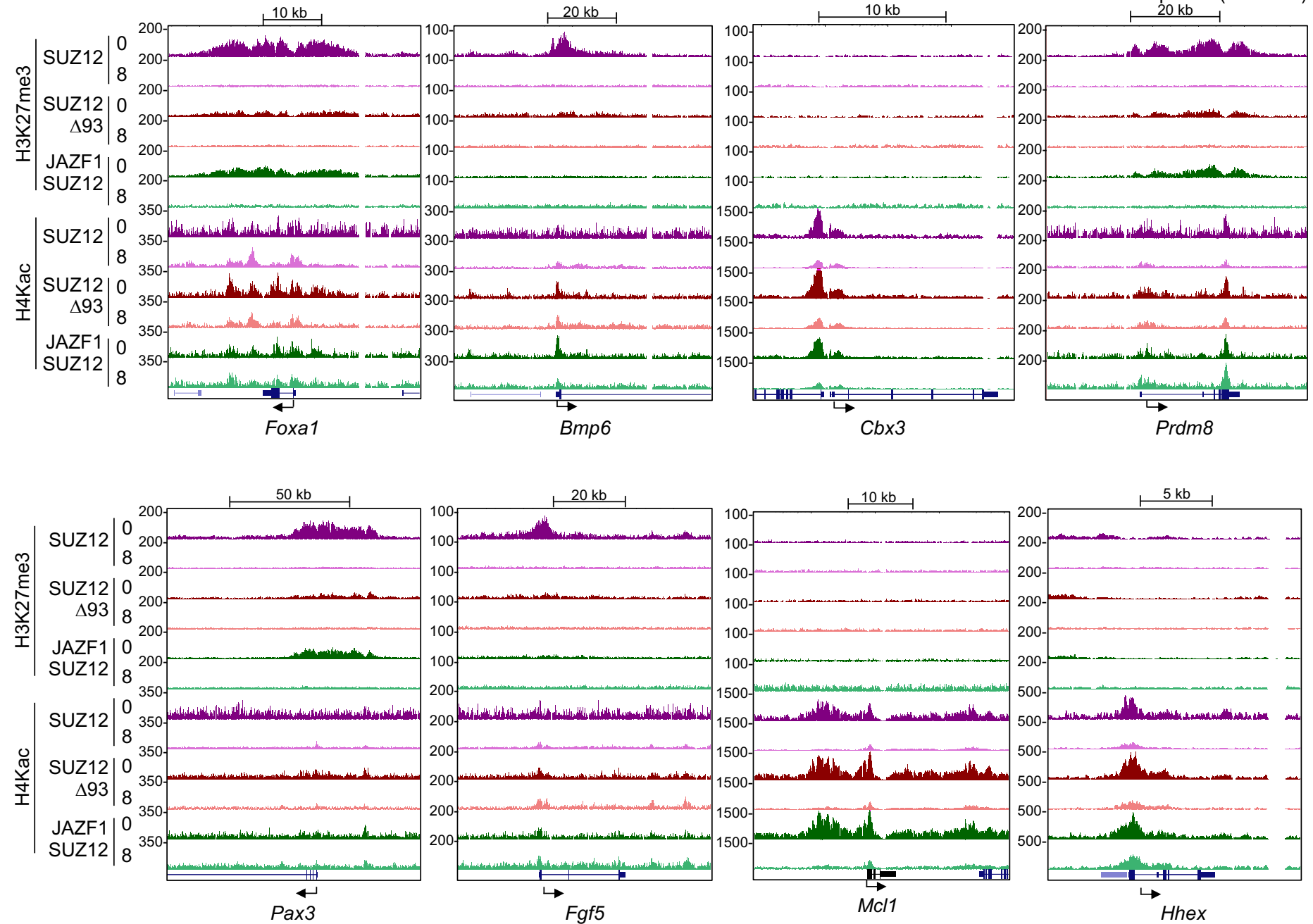

b

Shared JAZF1-SUZ12 and JAZF1 binding sites

ESC

SUZ12 JAZF1-

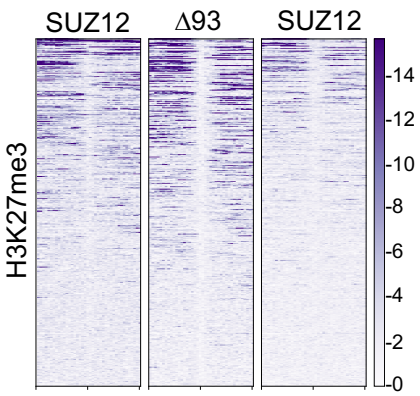

$\frac{\pi}{1}$

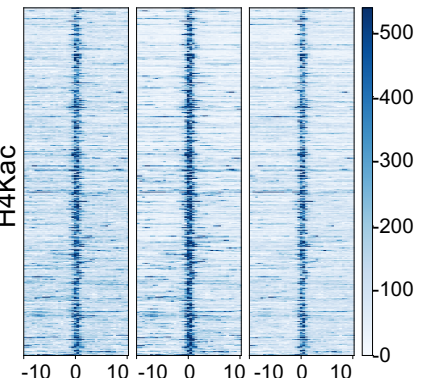

EB (8 days)

SUZ12 JAZF1-
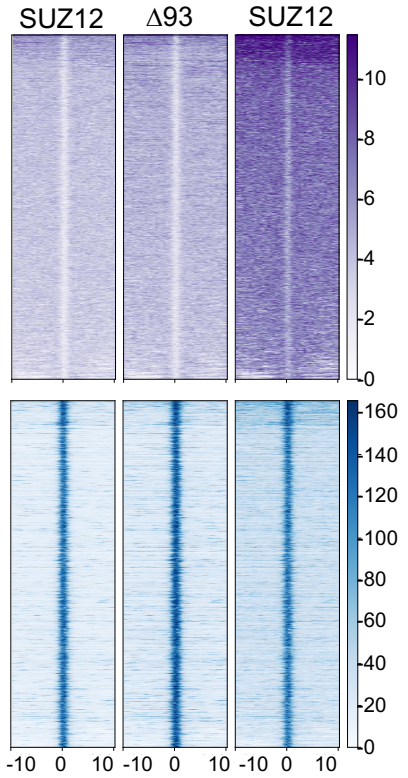

Figure S4. Changes in H3K27me3 and H4Kac in cells expressing JAZF1-SUZ12. a. H3K27me3 and H4Kac (reads per 10bp window) at the regions shown in Figure S3C in ESC expressing SUZ12, SUZ12 $\triangle 93$ or JAZF1-SUZ12 at days 0 and 8 after initiation of differentiation into EBs.

b. Heatmaps of H3K27me3 (purple, top) and $\mathrm{H} 4 \mathrm{Kac}$ (blue, bottom) around binding sites shared by JAZF1 and JAZF1-SUZ12 in ESC and in EB (day 8). Occupancy (normalised reads) is indicated by color, according to the scales on the right. At each timepoint, sites are ordered by H3K27me3 occupancy in cells expressing SUZ12, from high to low. 
a
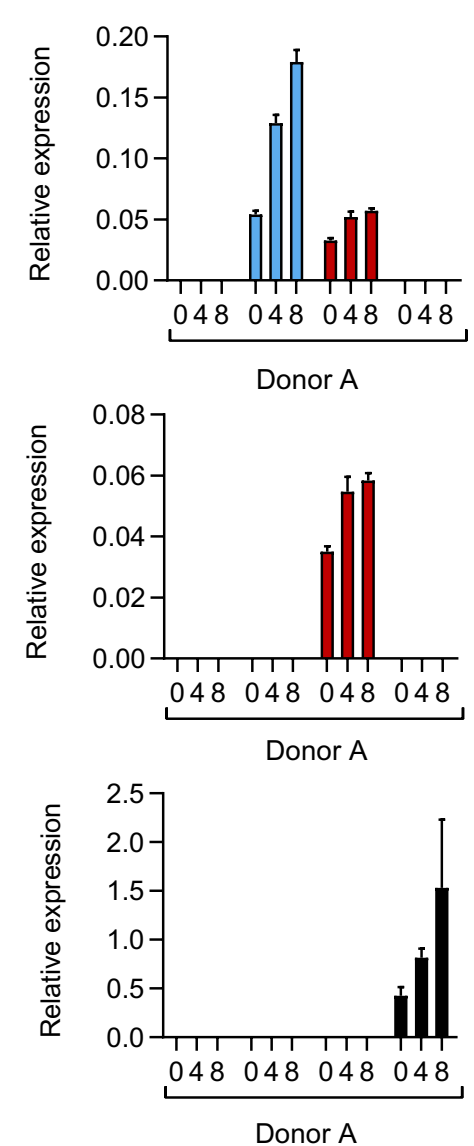

口JAZF1-SUZ12

口JAZF1

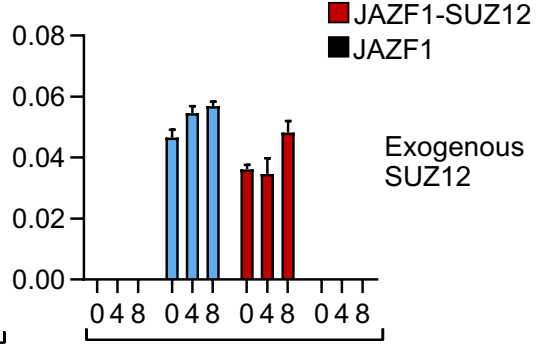

Donor B
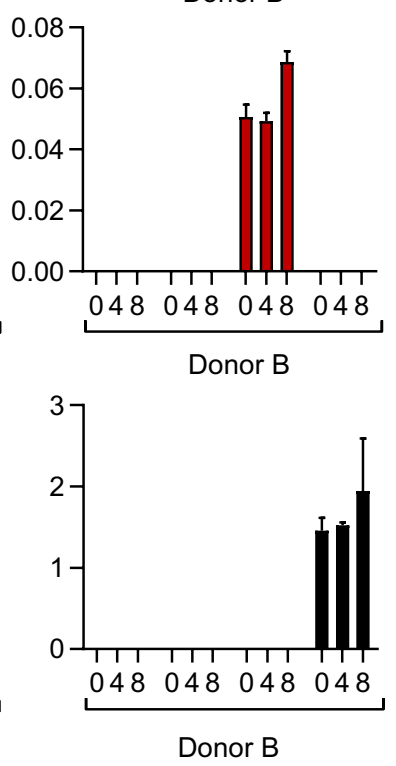

JAZF1-

SUZ12 JAZF1 b

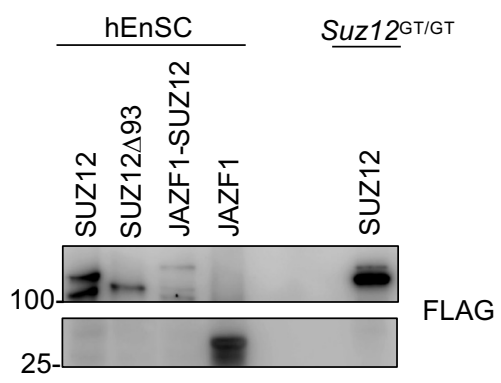

C

Exogenous
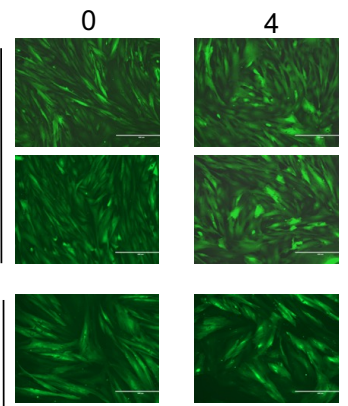

Donor B
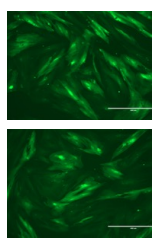

8
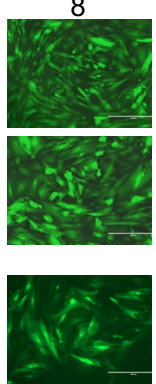

$\left[\begin{array}{c}2 y \\ -12\end{array}\right.$

Figure S5. Expression of transgenes in decidualised hEnSC.

a. Expression of exogenous SUZ12, JAZF1-SUZ12 and JAZF1 constructs relative to 5S rRNA at 0,4 and 8 days after initiating decidualisation of hEnSC from 2 donors.

b. Immunoblot for FLAG-tagged proteins in whole cell lysate of hEnSC stably expressing FLAGtagged SUZ12, SUZ12 493 , JAZF1-SUZ12 and JAZF1 in comparison to Suz12 ${ }^{\mathrm{GT} / G T}$ ESC stably expressing FLAG-tagged SUZ12.

c. Representative images of hEnSC from two donors expressing GFP-FLAG at 0,4 and 8 days after initiation of decidualisation with cAMP and MPA showing gain of an epithelioid phenotype. The scale bars are $400 \mu \mathrm{m}$. 